\title{
Bottleneck model revisited: An activity-based perspective
}

\author{
Zhi-Chun Li, ${ }^{\text {a } W i l l i a m ~ H . ~ K . ~ L a m, ~}{ }^{\text {b,c* }}$ S.C. Wong ${ }^{\mathrm{d}}$ \\ ${ }^{a}$ School of Management, Huazhong University of Science and Technology, Wuhan 430074, China \\ ${ }^{\mathrm{b}}$ Department of Civil and Environmental Engineering, The Hong Kong Polytechnic University, Kowloon, \\ Hong Kong, China \\ ${ }^{\mathrm{c}}$ School of Traffic and Transportation, Beijing Jiaotong University, Beijing 100044, China \\ ${ }^{\mathrm{d}}$ Department of Civil Engineering, The University of Hong Kong, Pokfulam Road, Hong Kong, China
}

\begin{abstract}
The timing of commuting trips made during morning and evening peaks has typically been investigated using Vickrey's bottleneck model. However, in the conventional trip-based approach, the decisions that commuters make during the day about their activity schedules and time use are not explicitly considered. This study extends the bottleneck model to address the scheduling problem of commuters' morning home-to-work and evening work-to-home journeys by using an activity-based approach. A day-long activity-travel scheduling model is proposed for the simultaneous determination of departure times for morning and evening commutes, together with allocations of time during the day among travel and activities undertaken at home or at the workplace. The proposed model maximizes the total net utility of the home-based tour, which is the difference between the benefits derived from participating in activities and the disutility incurred by travel between activity locations. The properties of the model solution are analytically explored and compared with the conventional bottleneck model for a special case with constant marginal-activity utility. For the case with linear marginal-activity utility, we develop a heuristic procedure to seek the equilibrium scheduling solution. We also explore the effects of marginal-work utility (or the employees' average wage level) and of flexible work-hour schemes on the scheduling problem in relation to the morning and evening commuting tours.
\end{abstract}

Keywords: Bottleneck model, tour, trip-based approach, activity-based approach, marginal-activity utility function, flexible work-hour scheme.

\footnotetext{
${ }^{*}$ Corresponding author. Tel.: +852-2766-6045; Fax: +852-2334-6389.

E-mail addresses: smzcli@hust.edu.cn (Z.-C. Li), cehklam@polyu.edu.hk (W.H.K. Lam), hhecwsc@hkucc.hku.hk (S.C. Wong).
} 


\section{Introduction}

The bottleneck model introduced by Vickrey (1969) has been recognized as a benchmark representation of the dynamics of peak-hour traffic congestion due to its ability to capture the essence of congestion dynamics in a simple and tractable way. The standard bottleneck model has been extended in numerous ways, such as by considering heterogeneous travelers (Arnott et al., 1988, 1992; Lindsey, 2004; Qian and Zhang, 2013; van den Berg, 2014), bottleneck congestion pricing (van den Berg and Verhoef, 2011, 2014; Xiao et al., 2011, 2012; Lindsey et al., 2012), bottleneck capacity expansion (Arnott et al., 1990; Arnott and Kraus, 1995), interaction between parallel or serial bottlenecks (Huang and Yang, 1996), mode substitution (Tabuchi, 1993; Huang, 2002; Huang et al., 2007; Gonzales and Daganzo, 2013), and recently, designing tradable credit schemes (Nie and Yin, 2013; Xiao et al., 2013) and considering stochastic bottleneck (Fosgerau and Lindsey, 2013; Siu and Lo, 2013; Xiao et al., 2014). Arnott et al. (1993) provided a structural definition of the bottleneck model and illustrated its application in the assessment of various congestion toll-pricing schemes. For a comprehensive review, readers can refer to Arnott et al. (1998) and Lindsey and Verhoef (2001).

Vickrey's bottleneck model and its variations usually treat a single-trip scheduling problem by modeling the trade-off between bottleneck congestion and schedule delay, and thus falls into the trip-based modeling framework. Trip-based models do not explicitly recognize the motivations or reasons for trips, and simply use discrete trips as the standard travel unit. Thus, they cannot reflect the links among trips, the links between trips and activities, and the temporal constraints and dependencies of participation in the activities concerned (Kitamura, 1988; Lam and Yin, 2001; Lam and Huang, 2002; Fu and Lam, 2014). As a result, the trip-based approach cannot properly capture the activity and travel-choice behavior of individuals or their allocations of time during a day among activities and travel, which may lead to biased or distorted evaluations of transport policy influence on individuals' activity-travel scheduling (Li et al., 2010).

In reality, the departure-time choices of commuters leaving home in the morning and their workplaces in the evening are usually related to the utilities of home and work activities in addition to the factors of bottleneck congestion and schedule delay (Zhang et al., 2005; Ettema et al., 2007; Jenelius et al., 2011; Jenelius, 2012). For example, when the home 
activity in the morning (e.g., preparing breakfast for the children) has a higher marginal utility than the work activity, the commuter may leave home late to achieve a high level of utility from participating in the home activity. Conversely, when the marginal utility of the work activity is higher than that of a home activity (e.g., a high overtime payment), the commuter may stay in the office for a longer time. In addition, when the home activity in the evening (e.g., family dinner, watching television or sleeping) has a higher marginal utility than the work activity, the commuter may leave the office early to perform more home activities in the evening. Hence, it is of great importance that the activity-scheduling behavior of the commuters is incorporated into the traditional bottleneck model, such that the effects of activity utility on the commuters' departure-time choices and time-use decisions can be revealed.

It has been widely recognized that the activity-based approach can serve as a powerful tool for understanding activity-travel scheduling behavior (Kitamura, 1988). Activity-based models consider travel as demand derived from the need to participate in activities at different points in space and time, and individuals' activity-travel patterns as the results of time-use decisions within a continuous time domain (e.g., a day). In activity-based models, travel patterns are organized as sets of related trips known as "tours." These tours are chains of multiple trips that begin and end at the same point, such as the commuter's home. Activity-based models can address the interdependencies of trips and activities in time and space and the time-use decisions that individuals make during the day. For more details on the activity-based approach, readers can refer to Jones et al. (1990), Ettema and Timmermans (1997), and Timmermans (2005). In this paper, we propose an activity-based bottleneck model to address the interactions between commuters' time allocations among their activities and travel and the dynamics of bottleneck congestion.

The standard bottleneck model also focuses mainly on morning commuting trips in which commuters are assumed to care about when they depart from their homes. Little attention has been paid to evening or day-long commuting problems. This may have arisen because the evening commuting trip is usually considered to be a symmetric reverse process of the morning trip. Some previous studies, such as those by Vickrey (1973), Hurdle (1981), Fargier (1983), and de Palma and Lindsey (2002a) have investigated morning and evening departure patterns in isolation and shown that the morning and evening departure patterns of specific individuals were symmetric (i.e., the departure pattern of the morning commute was a mirror 
image of that for the evening commute). However, this symmetry tends to break down with increased heterogeneity among commuters in terms of their preferred work start/end times, the value of their time and the costs of schedule delays. De Palma and Lindsey (2002b) illustrated this lack of symmetry in the presence of congestion toll pricing.

Although investigating the morning and evening commuting problems in isolation provides some important insights, in reality, commuters usually make travel decisions based on their day-long schedules. In the literature to date only a few published papers have involved analysis of day-long commuting problems. For example, Zhang et al. (2008) proposed an integrated daily commuting model that linked the morning and evening trips via choice of parking location. Recently, Gonzales and Daganzo (2013) incorporated mode choice in the combined morning and evening commute problem. More recently, Daganzo (2013) has further examined the day-long problem by considering two modes (auto and transit) and their distributed demand. However, the day-long commuting models developed in these studies have adopted the trip-based modeling approach, which means that the time-use decisions of commuters and the effects of flexibility in their activity scheduling cannot be properly addressed.

Zhang et al. (2005) presented a day-long activity-travel scheduling model to address commuters' time allocations among activities and travel during a day. Their model connected the morning and evening commutes via work duration. However, their paper did not examine the analytical properties of their proposed model. Recently, Jenelius et al. (2011) and Jenelius (2012) extended the Zhang et al. (2005) model to determine the values of travel-time savings and travel-time variability in both deterministic and stochastic environments. However, strong assumptions (e.g., no traffic congestion at the bottleneck) were made for derivation of some analytical properties of this model. In addition, none of these researchers (Zhang et al., Jenelius et al. or Jenelius) have considered the effects of schedule delays associated with work activities (which are usually mandatory activities). Recently, Ettema et al. (2007) showed that schedule delays for work activities play an important role in the commuters' scheduling of daily activities and travel, and such delays should therefore be considered in an activity-based model.

In light of the above discussion, we extend the traditional Vickrey bottleneck model to address commuters' time allocations among activities and travel during a day, and investigate the 
effects of home/work-activity utilities on the commuters' morning and evening departure patterns. The problems addressed here are defined as follows. Given a typical "home-work-home" activity chain, how does a worker/commuter allocate the time in a day (i.e., 24 hours) among activities and travel and choose the departure time of each activity such that his/her total net utility within a day is maximized? Is there a correlation between the commuters' morning and evening departure-time choices? Under what conditions do queues occur at the bottleneck during the morning and evening commuting trips? If queues exist during the morning and evening peaks, which queuing delay (or en-route travel time) is longer? If a flexible work-hour scheme is implemented, then how does it affect the time-allocation decisions of commuters during the day, and what is the optimal degree of flexibility for such a scheme?

To answer these important and interesting problems, an activity-based bottleneck model is proposed in this paper to model the trip-timing choices of commuters during their morning and evening commutes. The main contributions of this paper are as follows. First, an activity-based bottleneck model is proposed for simultaneous determination of the equilibrium departure-time choices for the morning and evening commutes. Compared to the traditional bottleneck model, the proposed model can endogenously determine the commuters' allocations of time among activities and travel during a day. Second, the properties of the activity-based bottleneck model are analytically explored and compared with those of the traditional bottleneck model, particularly when the marginal utilities of home/work activities are constants. A sufficient and necessary condition for the occurrence of queues at the bottleneck during the morning and evening peaks and that for the interdependence between morning and evening departure-time choices are presented. The effects of work-activity utility (i.e., the employees' average wage level) on queuing delays during the morning and evening peaks are also explored. Third, we examine the effects of flexibility in the work-hour scheme on commuters' activity-travel scheduling patterns and their time-use decisions during the day. The optimal level of work-hour flexibility in terms of total user net utility of the system during the day is also determined.

The remainder of this paper is organized as follows. In the next section, the activity-based bottleneck model is proposed. Section 3 formulates the properties of the proposed model and presents analytical solutions for a special case with constant marginal-activity utility. In Section 4, another case with linear marginal-activity utility is presented, together with a 
heuristic solution algorithm. In Section 5, two numerical examples are given to illustrate the properties and applications of the proposed model. Finally, Section 6 provides our conclusions and recommendations for further studies.

\section{Activity-based bottleneck model}

In this section, we extend the standard bottleneck model to address the activity-travel scheduling behavior of commuters over the times of a day. We consider a typical "home-work-home" (or "home-based tour") activity chain, as shown in Figure 1. Kawakami and Isobe's (1990) empirical study showed that the "home-work-home" chain is the most typical activity pattern for worker. It accounts for almost $90 \%$ of workers' activity patterns. Thus, we mainly focus on this typical home-based tour in this paper.

In Figure 1, it is assumed that every morning, $N$ commuters travel to workplace $\mathrm{W}$ for work, and then return home $\mathrm{H}$ every evening along the same route. This chain consists of three activities (i.e., home activity in the morning, work during the day and home activity in the evening) and a chain of two intermediate trips (i.e., travel from home to work in the morning and from work to home in the evening). To calculate the total utility of this tour, the disutility of a round journey between home and the workplace and the utilities of home and work activities are defined as follows.

\subsection{Disutility of travel}

The total disutility of travel on the "home-work-home" tour consists of the travel times from home to work in the morning and from work to home in the evening and the costs of schedule delays from arriving early or late at the workplace in the morning and departing early or late from the workplace in the evening.

\subsubsection{Travel time}

In Figure 1, the route connecting the commuter's home and workplace consists of three segments: HA, with a constant travel time of $T_{1}$; BW, with a constant travel time of $T_{2}$; and $\mathrm{AB}$, which is a bottleneck with a maximum service rate or capacity of $S$. When the arrival rate 
of vehicles at the bottleneck is less than $S$, the travel time on $\mathrm{AB}$ is a constant $T_{0}$. However, when the arrival rate exceeds $S$, a queue forms at the bottleneck. Let the subscripts " $M$ " and "E" represent the "morning" and "evening" trips, respectively. Let $T_{M}(t)$ and $T_{E}(t)$ be the waiting times at the bottleneck for a commuter departing from home in the morning and from work in the evening at time $t$, respectively. Thus, the travel times of commuters departing at time $t$ from home in the morning, $\Phi_{M}(t)$, and from work in the evening, $\Phi_{E}(t)$, can be respectively defined as

$$
\begin{aligned}
& \Phi_{M}(t)=T_{1}+T_{M}(t)+T_{0}+T_{2}=T_{f}+T_{M}(t), \text { and } \\
& \Phi_{E}(t)=T_{2}+T_{E}(t)+T_{0}+T_{1}=T_{f}+T_{E}(t),
\end{aligned}
$$

where $T_{f}$ is the fixed component of travel time between home and workplace, i.e., $T_{f}=T_{1}+T_{0}+T_{2}$. Without loss of generality, we set $T_{f}=0$ (i.e., zero free-flow travel time). This assumption, which has been made in the standard bottleneck model, does not affect the results that are of interest, and it can facilitate comparison with the results of the standard bottleneck model. Thus, a commuter arrives at the bottleneck immediately after leaving home and arrives at his/her workplace immediately after leaving the bottleneck. Hence, $\Phi_{M}(t)=T_{M}(t)$ and $\Phi_{E}(t)=T_{E}(t)$ hold, and thus $\Phi_{M}(t)$ and $T_{M}(t)$, and $\Phi_{E}(t)$ and $T_{E}(t)$ can be used interchangeably in this analysis.

Let $D_{M}(t)$ and $D_{E}(t)$ be the numbers of vehicles in the queues (i.e., queue lengths) at the bottleneck at time $t$ in the morning and evening peaks, respectively. When there is no congestion at the bottleneck, $D_{M}(t)=D_{E}(t)=0$, and the departure rate of the bottleneck equals the arrival rate. When a queue occurs, $D_{M}(t)>0$ and $D_{E}(t)>0$, and the departure rate of the bottleneck equals its capacity. The queue length at the bottleneck at any time equals the difference between cumulative arrivals and cumulative departures by that time, which is expressed as

$$
\begin{aligned}
& D_{M}(t)=\int_{\hat{t}_{M}}^{t} r_{M}(t) d t-S\left(t-\hat{t}_{M}\right) \quad \text { for the morning commute, and } \\
& D_{E}(t)=\int_{\hat{t}_{E}}^{t} r_{E}(t) d t-S\left(t-\hat{t}_{E}\right) \quad \text { for the evening commute, }
\end{aligned}
$$

where $\hat{t}_{M}$ and $\hat{t}_{E}$ are the most recent times at which there is no queue during the morning and evening peaks, respectively, and $r_{M}(t)$ and $r_{E}(t)$ are the departure rates for the morning and evening commuting trips at time $t$, respectively. 
The rates of change in the queue lengths can thus be given by

$$
\begin{aligned}
& \frac{d D_{M}(t)}{d t}=\left\{\begin{array}{l}
r_{M}(t)-S, \text { for } D_{M}(t)>0, \\
0, \text { otherwise, }
\end{array}\right. \text { for the morning commute, and } \\
& \frac{d D_{E}(t)}{d t}=\left\{\begin{array}{l}
r_{E}(t)-S, \text { for } D_{E}(t)>0, \\
0, \text { otherwise, }
\end{array}\right.
\end{aligned}
$$

The waiting times $T_{M}(t)$ and $T_{E}(t)$ at the bottleneck for a departure from home and departure from work at time $t$ can be derived from a deterministic queue model; that is, the queuing time of a commuter at the bottleneck equals the queue length at the time that he/she joins the queue divided by the service rate (or capacity) of the bottleneck. This can be expressed as

$$
\begin{aligned}
& T_{M}(t)=\frac{D_{M}(t)}{S}, \text { and } \\
& T_{E}(t)=\frac{D_{E}(t)}{S} .
\end{aligned}
$$

\subsubsection{Schedule delay}

Work activities are usually obligatory and have rigid time windows, i.e., there are usually strict work start (e.g., 09:00) and end (e.g., 17:00) times. This means that arriving at the office early or late in the morning and departing early or late in the evening invoke penalties (de Palma and Lindsey, 2002a,b). When a flexible work-hour scheme is implemented, the schedule delay caused by a deviation from the "core work-hour period" can be explained as an extra cost or loss due to a weakened synchrony among the employees' work hours. We define a schedule delay for the morning commute as the difference between the actual and preferred arrival times at the workplace, whereas a schedule delay for the evening commute is the difference between the actual and preferred departure times from the workplace. In general, assembly-line workers, clerks or support staff in white-collar jobs have a high schedule-delay value due to rigid work schedules.

We use the subscripts " $h$ " and " $w$ " to represent "home" and "work" and the superscripts " $d$ " and " $a$ " to represent "departure" and "arrival", respectively. Let $t_{w}^{a}$ and $t_{w}^{a^{*}}$ be the actual 
and the preferred arrival times at the workplace in the morning, respectively. For a commuter leaving home at time $t_{h}^{d}$ in the morning, his/her actual arrival time at workplace $t_{w}^{a}$ is $t_{h}^{d}+T_{M}\left(t_{h}^{d}\right)$. Thus, the time for early arrival is $\max \left(0, t_{w}^{a^{*}}-t_{w}^{a}\right)$ or $\max \left(0, t_{w}^{a^{*}}-t_{h}^{d}-T_{M}\left(t_{h}^{d}\right)\right)$, and the time for late arrival is $\max \left(0, t_{w}^{a}-t_{w}^{a^{*}}\right)$ or $\max \left(0, t_{h}^{d}+T_{M}\left(t_{h}^{d}\right)-t_{w}^{a^{*}}\right)$. Therefore, the schedule-delay cost of the morning commute for a commuter departing at time $t$ is

$$
S D_{M}=\beta \max \left(0, t_{w}^{a^{*}}-t_{h}^{d}-T_{M}\left(t_{h}^{d}\right)\right)+\gamma \max \left(0, t_{h}^{d}+T_{M}\left(t_{h}^{d}\right)-t_{w}^{a^{*}}\right),
$$

where $\beta$ is the unit cost of arriving early, and $\gamma$ is the unit cost of arriving late.

Let $t_{w}^{d}$ and $t_{w}^{d^{*}}$ be the actual and the preferred departure times from the workplace in the evening, respectively. The early and late departure times are $\max \left(0, t_{w}^{d^{*}}-t_{w}^{d}\right)$ and $\max \left(0, t_{w}^{d}-t_{w}^{d^{*}}\right)$, respectively. The schedule-delay cost of the evening commute for a commuter departing at time $t$ is thus defined as

$$
S D_{E}=\mu \max \left(0, t_{w}^{d^{*}}-t_{w}^{d}\right)+\lambda \max \left(0, t_{w}^{d}-t_{w}^{d^{*}}\right),
$$

where $\mu$ is the unit cost of departing early, and $\lambda$ is the unit cost of departing late.

\subsubsection{Disutility of travel}

As previously stated, the total disutility of travel on the home-work-home tour, denoted as $U^{T}$, is the sum of the round-journey times between home and workplace, and the schedule-delay costs of the morning and evening commutes. It can be expressed as

$$
\begin{aligned}
U^{T} & =\alpha T_{M}\left(t_{h}^{d}\right)+\beta \max \left(0, t_{w}^{a^{*}}-t_{h}^{d}-T_{M}\left(t_{h}^{d}\right)\right)+\gamma \max \left(0, t_{h}^{d}+T_{M}\left(t_{h}^{d}\right)-t_{w}^{a^{*}}\right) \\
& +\alpha T_{E}\left(t_{w}^{d}\right)+\mu \max \left(0, t_{w}^{d^{*}}-t_{w}^{d}\right)+\lambda \max \left(0, t_{w}^{d}-t_{w}^{d^{*}}\right),
\end{aligned}
$$

where $\alpha$ is the unit cost of travel time. Following previous related studies (see, e.g., Small, 1982; de Palma and Lindsey, 2002a,b), it is assumed that $\gamma>\alpha>\beta$ for the morning commuting trip, and $\mu>\alpha>\lambda$ for the evening trip.

\subsection{Utility of activity}

The activities involved in the home-based tour (i.e., home-work-home) consist of the morning home activity, work activity during the day, and the evening home activity. The utility 
achieved by a commuter performing an activity depends on what time the activity starts (in terms of clock time) and the activity's duration (Wang, 1996; Ettema and Timmermans, 2003; Ashiru et al., 2004; Zhang et al., 2005; Ettema et al., 2007; Li et al., 2010; Jenelius et al., 2011; Jenelius, 2012). We assume that a day's schedule is independent of preceding and subsequent days. We can thus fix two times, $t=0$ and $t=24$, that represent the start and the end of a day, respectively. Thus, there is no need to distinguish between time-of-day and duration-dependent utility for the morning and evening home activities. We denote $u_{h}(t)$ and $\widehat{u}_{h}(t)$ as the marginal utilities of the morning home activity and the evening home activity at time $t$, respectively. The marginal utility of the work activity depends on time $t$ of a day and the work duration $\left(t-t_{w}^{a}\right)$. According to Ettema and Timmermans (2003), the marginal utility of a work activity can be measured by a linear combination of time $t$ and the work duration $\left(t-t_{w}^{a}\right)$, namely $u_{w}\left((1-\xi) t+\xi\left(t-t_{w}^{a}\right)\right) \quad\left(\right.$ or $\left.u_{w}\left(t-\xi t_{w}^{a}\right)\right)$, where $\xi \in[0,1]$ is a parameter representing the flexibility in scheduling the work activity. " $\xi=0$ " implies that the marginal utility of the work activity depends entirely on the (clock) time of day, resulting in a rigid work-hour scheme. " $\xi=1$ " implies that the marginal utility of the work activity depends only on a required work duration, leading to a totally flexible work-hour scheme. This method has been adopted by some previous related studies, such as Zhang et al. (2005), Jenelius et al. (2011), and Jenelius (2012).

The total utility of all the activities performed by a commuter within a day, denoted as $U^{A}$, can then be represented as

$$
\begin{aligned}
U^{A} & =\int_{0}^{t_{h}^{d}} u_{h}(t) d t+\int_{t_{w}^{a}}^{t_{w}^{d}} u_{w}\left(t-\xi t_{w}^{a}\right) d t+\int_{t_{h}^{a}}^{24} \widehat{u}_{h}(t) d t \\
& =\int_{0}^{t_{h}^{d}} u_{h}(t) d t+\int_{t_{h}^{d}+T_{M}\left(t_{h}^{d}\right)}^{t_{w}^{d}} u_{w}\left(t-\xi\left(t_{h}^{d}+T_{M}\left(t_{h}^{d}\right)\right)\right) d t+\int_{t_{w}^{d}+T_{E}\left(t_{w}^{d}\right)}^{24} \widehat{u}_{h}(t) d t,
\end{aligned}
$$

where $t_{h}^{a}$ is the actual arrival time at home in the evening, $t_{h}^{a}=t_{w}^{d}+T_{E}\left(t_{w}^{d}\right)$.

\subsection{Total net utility of the home-based tour}

The total net utility $U$ of the home-based tour for a commuter departing from home at time $t_{h}^{d}$ in the morning and from the workplace at time $t_{w}^{d}$ in the evening is the difference of the total utility of the activity minus the total disutility of travel on this tour. It can be represented as 


$$
\begin{aligned}
& U\left(t_{h}^{d}, t_{w}^{d}\right)=U^{A}-U^{T} \\
& \quad=\int_{0}^{t_{h}^{d}} u_{h}(t) d t+\int_{t_{h}^{d}+T_{M}\left(t_{h}^{d}\right)}^{t_{w}^{d}} u_{w}\left(t-\xi\left(t_{h}^{d}+T_{M}\left(t_{h}^{d}\right)\right)\right) d t+\int_{t_{w}^{d}+T_{E}\left(t_{w}^{d}\right)}^{24} \widehat{u}_{h}(t) d t-\alpha\left(T_{M}\left(t_{h}^{d}\right)+T_{E}\left(t_{w}^{d}\right)\right) \\
& \quad-\beta \max \left(0, t_{w}^{a^{*}}-t_{h}^{d}-T_{M}\left(t_{h}^{d}\right)\right)-\gamma \max \left(0, t_{h}^{d}+T_{M}\left(t_{h}^{d}\right)-t_{w}^{a^{*}}\right) \\
& \quad-\mu \max \left(0, t_{w}^{d^{*}}-t_{w}^{d}\right)-\lambda \max \left(0, t_{w}^{d}-t_{w}^{d^{*}}\right) .
\end{aligned}
$$

For illustration purpose, we consider a constant marginal activity utility case, in which the marginal home/work activity utilities are constants (i.e., $u_{h}(t)=u_{h}, u_{w}(t)=u_{w}$, and $\left.\widehat{u}_{h}(t)=\widehat{u}_{h}\right)$. We assume that the commuter, who departs from home at $t_{h}^{d}$ in the morning and from work at $t_{w}^{d}$ in the evening, arrives at work early in the morning, leaves work early in the evening, and returns home after $t_{w}^{d^{*}}$. In an ideal situation, the commuter leaves home at $t_{w}^{a^{*}}$, arrives at work immediately at $t_{w}^{a^{*}}$, leaves work at $t_{w}^{d^{*}}$, and arrives home immediately at $t_{w}^{d^{*}}$. We can determine the commuter's loss of utility with regard to this ideal situation, which is shaded in Figure 2. Note that arriving early at work in the morning implies an increased work duration of $\left(t_{w}^{a^{*}}-t_{w}^{a}\right)$ and a decreased home activity duration of $\left(t_{w}^{a^{*}}-t_{h}^{d}\right)$, which lead to a gain in utility by $\left(u_{w}-\beta\right)\left(t_{w}^{a^{*}}-t_{w}^{a}\right)$ and a loss of utility by $u_{h}\left(t_{w}^{a^{*}}-t_{h}^{d}\right)$, respectively. Similarly, leaving work early in the evening, and returns home after $t_{w}^{d^{*}}$ imply a decreased work duration of $\left(t_{w}^{d^{*}}-t_{w}^{d}\right)$ and a decreased home activity duration of $\left(t_{h}^{a}-t_{w}^{d^{*}}\right)$, which are associated with a loss of utility by $u_{w}\left(t_{w}^{d^{*}}-t_{w}^{d}\right)$ and by $\widehat{u}_{h}\left(t_{h}^{a}-t_{w}^{d^{*}}\right)$, respectively. Moreover, an additional penalty by $\mu\left(t_{w}^{d^{*}}-t_{w}^{d}\right)$ due to early departure from work is incurred.

\subsection{Travel and activity-scheduling model}

Given Equation (13), the total net utility $U$ is a function of the departure time $t_{h}^{d}$ from home in the morning, the departure time $t_{w}^{d}$ from work in the evening, and the two travel times $T_{M}\left(t_{h}^{d}\right)$ and $T_{E}\left(t_{w}^{d}\right)$ between home and workplace in the morning and evening, which are functions of $t_{h}^{d}$ and $t_{w}^{d}$, respectively. Thus, once the departure times $t_{h}^{d}$ and $t_{w}^{d}$ are determined for a commuter, his/her total net utility $U$ can be determined. The activity-travel 
scheduling model that maximizes the total net utility of the home-based tour can thus be formulated as

$$
\max _{t_{h}^{d}, t_{w}^{d}} U\left(t_{h}^{d}, t_{w}^{d}\right),
$$

where the departure times $t_{h}^{d}$ and $t_{w}^{d}$ of commuters are the decision variables.

According to Equation (14), the activity-travel scheduling model is actually equivalent to a departure-time choice equilibrium problem. The solution to this problem is a pure-strategy Nash equilibrium with the departure times $t_{h}^{d}$ and $t_{w}^{d}$ as the strategy variables, which is defined as follows.

Definition 1. At equilibrium, any used combination of $t_{h}^{d}$ and $t_{w}^{d}$ yields equal net utility.

Definition 1 implies that when the equilibrium state is reached, all commuters choosing any departure-time pair or combination $\left(t_{h}^{d}, t_{w}^{d}\right)$ share an identical total net utility, and no one can increase his/her total net utility over the course of a day by changing his/her departure time for the morning and/or evening commutes.

Let $q\left(t_{h}^{d}, t_{w}^{d}\right)$ be the departure rate of commuters choosing combination $\left(t_{h}^{d}, t_{w}^{d}\right)$. The equilibrium condition defined above can then be expressed as a complementarity problem with respect to the departure rate $q\left(t_{h}^{d}, t_{w}^{d}\right)$, i.e.,

$$
\left\{\begin{array}{l}
q\left(t_{h}^{d}, t_{w}^{d}\right)\left[U\left(t_{h}^{d}, t_{w}^{d}\right)-U^{*}\right]=0, \\
q\left(t_{h}^{d}, t_{w}^{d}\right) \geq 0, U\left(t_{h}^{d}, t_{w}^{d}\right)-U^{*} \geq 0, \quad \forall t_{h}^{d}, t_{w}^{d},
\end{array}\right.
$$

where $U^{*}$ is the equilibrium (maximum) net utility received by commuters during a day.

Given the departure pattern $q\left(t_{h}^{d}, t_{w}^{d}\right)$, both the home-to-work departure rate $r_{M}\left(t_{h}^{d}\right)$ at time $t_{h}^{d}$ in the morning and the work-to-home departure rate $r_{E}\left(t_{w}^{d}\right)$ at time $t_{w}^{d}$ in the evening can be respectively calculated by

$$
r_{M}\left(t_{h}^{d}\right)=\int_{t_{E}^{b}}^{t_{E}^{e}} q\left(t_{h}^{d}, t\right) d t, \quad \forall t_{h}^{d}, \text { and }
$$




$$
r_{E}\left(t_{w}^{d}\right)=\int_{t_{M}^{b}}^{t_{M}^{e}} q\left(t, t_{w}^{d}\right) d t, \quad \forall t_{w}^{d},
$$

where $t_{M}^{b}$ and $t_{M}^{e}$ are the times at which the first and last commuters depart from home during the morning commute to work, respectively. $t_{E}^{b}$ and $t_{E}^{e}$ are the times at which the first and last commuters depart from the workplace during the evening commute to their homes, respectively.

To summarize, the travel and activity scheduling model (14) serves to find the equilibrium departure pattern $q\left(t_{h}^{d}, t_{w}^{d}\right)$ or, equivalently, the equilibrium departure rates $r_{M}\left(t_{h}^{d}\right)$ and $r_{E}\left(t_{w}^{d}\right)$ during the morning and evening commuting trips. By using this proposed model, the commuters' average time allocation among activities and travel can also be determined. In the next section, it will be shown that one can derive a closed-form solution for the equilibrium departure rates $r_{M}\left(t_{h}^{d}\right)$ and $r_{E}\left(t_{w}^{d}\right)$ for a special case with constant marginal-activity utility, but a numerical approach must be used for general cases, including those with linear marginal-activity utility.

\section{Equilibrium properties}

We first present an important property of the proposed model, namely a sufficient and necessary condition that a queue must exist at the bottleneck during the morning and evening commutes. Let $t_{M}^{o}$ denote the morning departure time from home at which a commuter can arrive at the workplace on time, i.e., $t_{M}^{o}+T_{M}\left(t_{M}^{o}\right)=t_{w}^{a^{*}}$. We make the following proposition.

Proposition 1. (i) Given the departure time $t_{w}^{d}$ from the workplace in the evening, a queue must exist at the bottleneck at time $t$ during the morning home-to-work commute if and only if the following conditions are satisfied:

$$
\left\{\begin{array}{l}
u_{h}(t)>\xi u_{w}\left(t_{w}^{d}-\xi t\right)-(\xi-1) u_{w}(t-\xi t)-\beta, \text { for } t \in\left(t_{M}^{b}, t_{M}^{o}\right], \\
u_{h}(t)<\xi u_{w}\left(t_{w}^{d}-\xi t\right)-(\xi-1) u_{w}(t-\xi t)+\gamma, \text { for } t \in\left[t_{M}^{o}, t_{M}^{e}\right) .
\end{array}\right.
$$

(ii) Given the arrival time $t_{w}^{a}$ at the workplace in the morning, a queue must exist at the bottleneck at time $t$ during the evening work-to-home commute if and only if the following 
conditions are satisfied:

$$
\left\{\begin{array}{l}
\widehat{u}_{h}(t)<u_{w}\left(t-\xi t_{w}^{a}\right)+\mu, \text { for } t \in\left(t_{E}^{b}, t_{w}^{d^{*}}\right], \\
\widehat{u}_{h}(t)>u_{w}\left(t-\xi t_{w}^{a}\right)-\lambda, \text { for } t \in\left[t_{w}^{d^{*}}, t_{E}^{e}\right) .
\end{array}\right.
$$

The proof of Proposition 1 is given in Appendix A. It shows that when the conditions (18) and (19) are not satisfied, a queue never occurs at the bottleneck during the morning or evening commutes. In particular, when the marginal utility functions of the home/work activities are constants (i.e., $u_{h}(t)=u_{h}, u_{w}(t)=u_{w}$, and $\widehat{u}_{h}(t)=\widehat{u}_{h}$ ), we have the following properties

Corollary 1. For a constant marginal-activity utility,

(i) If $-\beta<u_{h}-u_{w}<\gamma$ holds, then a queue must exist at the bottleneck during the morning peak $\left(t_{M}^{b}, t_{M}^{e}\right)$. If $-\lambda<\widehat{u}_{h}-u_{w}<\mu$ holds, then a queue must exist at the bottleneck during the evening peak $\left(t_{E}^{b}, t_{E}^{e}\right)$.

(ii) If $u_{h}-u_{w}+\beta \leq 0$ (or $u_{h}-u_{w}-\gamma \geq 0$ ), then all commuters leave home early (or late), and no queue occurs at the bottleneck during the morning peak. If $\widehat{u}_{h}-u_{w}-\mu \geq 0$ (or $\widehat{u}_{h}-u_{w}+\lambda \leq 0$ ), then all commuters leave the office early (or late) and no queue occurs at the bottleneck during the evening peak.

Proof. Part (i) can be directly obtained from Proposition 1. In the following, we prove the first part of (ii), that is, if $u_{h} \leq u_{w}-\beta$ or $u_{h} \geq u_{w}+\gamma$, a queue never occurs at the bottleneck during the morning peak. In fact, if $u_{h} \leq u_{w}-\beta$, then the marginal benefit of prolonging the work duration by one unit of time exceeds the sum of the marginal loss from shortening the home activity duration by one unit of time and the schedule-change cost due to early arrival of one unit of time. Hence, early departure from home can bring a positive net benefit due to an extended work duration (shaded area in Figure 3a). Under these conditions, all commuters would benefit by leaving home early.

However, if $u_{h} \geq u_{w}+\gamma$, then all commuters would benefit by departing late from home, because the marginal benefit caused by increasing the duration of the home activity by one unit of time (shaded area in Figure $3 b$ ) is not less than the sum of the marginal benefit from decreasing the duration of the work activity by one unit of time and the schedule-delay cost of 
late arrival of one unit of time. This condition would indicate that late departure from home can lead to a positive net benefit. Similarly, one can prove the latter part of (ii).

Remark 1. Corollary 1 shows that the trade-off between the utilities of home and work activities and the schedule delays involved can significantly affect the departure-time choices of commuters. Hence, it is of great importance to incorporate the commuters' activity-scheduling behavior into the bottleneck model to analyze the departure-time decisions of commuters. This incorporation cannot be achieved in the traditional trip-based bottleneck models. In addition, it can be noted that the traditional trip-based bottleneck models (i.e. the Vickrey's bottleneck model and its variations) satisfy the conditions in part (i) of Corollary 1. Consequently, a queue always exists in the traditional trip-based bottleneck models.

In the following proposition, we describe the interrelationship between the departure-time decisions of commuters for their morning and evening commuting trips.

Proposition 2. The commuters' morning and evening departure-time choices are interdependent if and only if the marginal utility of their work activity $u_{w}\left(t-\xi t_{w}^{a}\right)$ is not a constant, and $\xi \neq 0$.

Proof. The first-order partial derivatives of total net utility $U(\cdot)$ from Equation (14), with regard to the decision variables $t_{h}^{d}$ and $t_{w}^{d}$, comprise the following system of equations:

$$
\begin{aligned}
& \frac{\partial U(\cdot)}{\partial t_{h}^{d}}=\left\{\begin{array}{l}
u_{h}\left(t_{h}^{d}\right)-\xi u_{w}\left(t_{w}^{d}-\xi t_{w}^{a}\right)+(\xi-1) u_{w}\left(t_{w}^{a}-\xi t_{w}^{a}\right)+\beta-\left(\xi u_{w}\left(t_{w}^{d}-\xi t_{w}^{a}\right)+(1-\xi) u_{w}\left(t_{w}^{a}-\xi t_{w}^{a}\right)+\alpha-\beta\right) \frac{\partial T_{M}\left(t_{h}^{d}\right)}{\partial t_{h}^{d}}, \\
u_{h}\left(t_{h}^{d}\right)-\xi u_{w}\left(t_{w}^{d}-\xi t_{w}^{a}\right)+(\xi-1) u_{w}\left(t_{w}^{a}-\xi t_{w}^{a}\right)-\gamma-\left(\xi u_{w}\left(t_{w}^{d}-\xi t_{w}^{a}\right)+(1-\xi) u_{w}\left(t_{w}^{a}-\xi t_{w}^{a}\right)+\alpha+\gamma\right) \frac{\partial T_{M}\left(t_{h}^{d}\right)}{\partial t_{h}^{d}}, \quad \forall t_{h}^{d} \in\left[t_{M}^{o}, t_{M}^{e}\right] .
\end{array}\right. \\
& \frac{\partial U(\cdot)}{\partial t_{w}^{d}}= \begin{cases}u_{w}\left(t_{w}^{d}-\xi t_{w}^{a}\right)-\widehat{u}_{h}\left(t_{h}^{a}\right)+\mu-\left(\widehat{u}_{h}\left(t_{h}^{a}\right)+\alpha\right) \frac{\partial T_{E}\left(t_{w}^{d}\right)}{\partial t_{w}^{d}}, & \forall t_{w}^{d} \in\left[t_{E}^{b}, t_{w}^{d^{*}}\right], \\
u_{w}\left(t_{w}^{d}-\xi t_{w}^{a}\right)-\widehat{u}_{h}\left(t_{h}^{a}\right)-\lambda-\left(\widehat{u}_{h}\left(t_{h}^{a}\right)+\alpha\right) \frac{\partial T_{E}\left(t_{w}^{d}\right)}{\partial t_{w}^{d}}, & \forall t_{w}^{d} \in\left[t_{w}^{d^{*}}, t_{E}^{e}\right] .\end{cases}
\end{aligned}
$$

From Equation (20) or (21), we can obtain

$$
\frac{\partial U^{2}(\cdot)}{\partial t_{h}^{d} \partial t_{w}^{d}}=-\xi u_{w}^{\prime}\left(t_{w}^{d}-\xi t_{w}^{a}\right)\left(1+\frac{\partial T_{M}\left(t_{h}^{d}\right)}{\partial t_{h}^{d}}\right) .
$$


The independence of the morning and evening departure-time decisions means that the marginal contributions of $t_{h}^{d}$ and $t_{w}^{d}$ to the total net utility $U(\cdot)$ have no interaction, which requires Equation (22) to be 0 . Thus, $u_{w}\left(t-\xi t_{w}^{a}\right)$ is a constant, or $\xi=0$. Therefore, the morning and evening departure-time decisions are interdependent if and only if $u_{w}\left(t-\xi t_{w}^{a}\right)$ is not a constant, and $\xi \neq 0$. This completes the proof of this proposition.

Remark 2. It can be noted that there is generally no closed-form solution for the morning and evening queuing delays $T_{M}\left(t_{h}^{d}\right)$ and $T_{E}\left(t_{w}^{d}\right)$, except for the no bottleneck congestion case (see Jenelius et al., 2011) and the constant marginal activity utility case (as shown in Corollary 2 as below). Therefore, the concavity of the Hessian matrix of the net utility function $U\left(t_{h}^{d}, t_{w}^{d}\right)$, and thus the uniqueness of the model solution, cannot be guaranteed.

According to Proposition 2, one immediately obtains the following.

Corollary 2. For a constant marginal-activity utility, the commuters' departure-time choices in the morning and evening periods are independent.

For the constant marginal-activity utility case, one can determine the unique optimal solution of the activity-travel scheduling model (14) as given below. According to the extreme value theory of multivariate functions, the critical points of the activity-travel scheduling model (14) with regard to the decision variables $t_{h}^{d}$ and $t_{w}^{d}$ require that Equations (20) and (21) both be zero, i.e., $\partial U(\cdot) / \partial t_{h}^{d}=0$ and $\partial U(\cdot) / \partial t_{w}^{d}=0$. When the marginal utilities of all activities are constants, i.e., $u_{h}(t)=u_{h}, u_{w}(t)=u_{w}$ and $\widehat{u}_{h}(t)=\widehat{u}_{h}$, one can obtain from Equations (20) and (21)

$$
\begin{aligned}
& \left\{\begin{array}{l}
u_{h}-u_{w}+\beta-\left(u_{w}+\alpha-\beta\right) \frac{\partial T_{M}\left(t_{h}^{d}\right)}{\partial t_{h}^{d}}=0, \quad \forall t_{h}^{d} \in\left[t_{M}^{b}, t_{M}^{o}\right], \\
u_{h}-u_{w}-\gamma-\left(u_{w}+\alpha+\gamma\right) \frac{\partial T_{M}\left(t_{h}^{d}\right)}{\partial t_{h}^{d}}=0, \quad \forall t_{h}^{d} \in\left[t_{M}^{o}, t_{M}^{e}\right],
\end{array}\right. \\
& \left\{\begin{array}{l}
u_{w}-\widehat{u}_{h}+\mu-\left(\hat{u}_{h}+\alpha\right) \frac{\partial T_{E}\left(t_{w}^{d}\right)}{\partial t_{w}^{d}}=0, \quad \forall t_{w}^{d} \in\left[t_{E}^{b}, t_{w}^{d^{*}}\right], \\
u_{w}-\widehat{u}_{h}-\lambda-\left(\hat{u}_{h}+\alpha\right) \frac{\partial T_{E}\left(t_{w}^{d}\right)}{\partial t_{w}^{d}}=0, \quad \forall t_{w}^{d} \in\left[t_{w}^{d *}, t_{E}^{e}\right],
\end{array}\right.
\end{aligned}
$$


From Equations (23) and (24), we obtain

$$
\begin{aligned}
& \frac{\partial T_{M}\left(t_{h}^{d}\right)}{\partial t_{h}^{d}}=\left\{\begin{array}{ll}
\frac{u_{h}-u_{w}+\beta}{u_{w}+\alpha-\beta}, & \forall t_{h}^{d} \in\left[t_{M}^{b}, t_{M}^{o}\right], \\
\frac{u_{h}-u_{w}-\gamma}{u_{w}+\alpha+\gamma}, & \forall t_{h}^{d} \in\left[t_{M}^{o}, t_{M}^{e}\right],
\end{array}\right. \text { for the morning commute, and } \\
& \frac{\partial T_{E}\left(t_{w}^{d}\right)}{\partial t_{w}^{d}}= \begin{cases}\frac{u_{w}-\widehat{u}_{h}+\mu}{\widehat{u}_{h}+\alpha}, & \forall t_{w}^{d} \in\left[t_{E}^{b}, t_{w}^{d^{*}}\right], \\
\frac{u_{w}-\widehat{u}_{h}-\lambda}{\widehat{u}_{h}+\alpha}, & \forall t_{w}^{d} \in\left[t_{w}^{d^{*}}, t_{E}^{e}\right],\end{cases}
\end{aligned}
$$

Combining Equations (25), (26) and (5)-(8), we obtain the equilibrium departure rates of the morning and evening peaks as follows:

$$
\begin{aligned}
& r_{M}\left(t_{h}^{d}\right)=\left\{\begin{array}{ll}
\frac{\alpha+u_{h}}{\alpha-\beta+u_{w}} S, & \forall t_{h}^{d} \in\left[t_{M}^{b}, t_{M}^{o}\right], \\
\frac{\alpha+u_{h}}{\alpha+\gamma+u_{w}} S, & \forall t_{h}^{d} \in\left[t_{M}^{o}, t_{M}^{e}\right],
\end{array}\right. \text { for the morning commute, and } \\
& r_{E}\left(t_{w}^{d}\right)=\left\{\begin{array}{ll}
\frac{\alpha+\mu+u_{w}}{\alpha+\widehat{u}_{h}} S, & \forall t_{w}^{d} \in\left[t_{E}^{b}, t_{w}^{d^{*}}\right], \\
\frac{\alpha-\lambda+u_{w}}{\alpha+\widehat{u}_{h}} S, & \forall t_{w}^{d} \in\left[t_{w}^{d^{*}}, t_{E}^{e}\right],
\end{array}\right. \text { for the evening commute. }
\end{aligned}
$$

Equations (27) and (28) show that the equilibrium departure rates under the conditions of constant marginal-activity utility are constants. This constancy means that the commuters' departure-time choice decisions in the morning and evening are uncorrelated.

Remark 3. The independence of the morning and evening departure-time choice decisions implies that at the equilibrium, the first individual to leave home in the morning can be the last to leave work in the evening, and the last individual to leave home in the morning can be the first to leave work in the evening. In addition, according to Equations (27) and (28) and Corollary 1 , when $-\beta<u_{h}-u_{w}<\gamma$ is satisfied for the morning commute, the equilibrium departure rate during the early arrival period $\left[t_{M}^{b}, t_{M}^{o}\right]$ exceeds the capacity $S$ of the bottleneck, whereas the equilibrium departure rate during the late arrival period $\left[t_{M}^{o}, t_{M}^{e}\right]$ is less than $S$. This condition means that in the morning peak, a queue builds up linearly from $t_{M}^{b}$ to $t_{M}^{o}$ and then dissipates linearly until it disappears at $t_{M}^{e}$. Similarly, when 
$-\lambda<\widehat{u}_{h}-u_{w}<\mu$ holds for the evening commute, a queue grows linearly from $t_{E}^{b}$ to $t_{w}^{d^{*}}$, reaches its maximum at $t_{w}^{d^{*}}$, and then shrinks linearly to zero at $t_{E}^{e}$.

We now determine the equilibrium departure periods $\left[t_{M}^{b}, t_{M}^{e}\right]$ and $\left[t_{E}^{b}, t_{E}^{e}\right]$. As stated above, when $-\beta<u_{h}-u_{w}<\gamma$ and $-\lambda<\widehat{u}_{h}-u_{w}<\mu$, a queue exists in the periods $\left(t_{M}^{b}, t_{M}^{e}\right)$ and $\left(t_{E}^{b}, t_{E}^{e}\right)$, respectively. Therefore, the departure rates from the bottleneck during the morning and evening peaks equal the capacity of the bottleneck. Thus, we have

$$
\left\{\begin{array}{l}
t_{M}^{e}-t_{M}^{b}=N / S \\
t_{E}^{e}-t_{E}^{b}=N / S
\end{array}\right.
$$

At the equilibrium, however, the commuters who depart at the beginnings and ends of the departure periods $\left[t_{M}^{b}, t_{M}^{e}\right]$ and $\left[t_{E}^{b}, t_{E}^{e}\right]$ face no queue at the bottleneck, but will incur the highest schedule-delay costs. Their total net utilities must be equal in terms of Definition 1; that is,

$$
\left\{\begin{array}{l}
U\left(t_{M}^{b}, t_{w}^{d}\right)=U\left(t_{M}^{e}, t_{w}^{d}\right), \quad \forall t_{w}^{d}, \\
U\left(t_{h}^{d}, t_{E}^{b}\right)=U\left(t_{h}^{d}, t_{E}^{e}\right), \quad \forall t_{h}^{d} .
\end{array}\right.
$$

By substituting Equation (13) into (30) and solving the system of Equations (29) and (30), we can obtain the equilibrium departure times, $t_{M}^{b}, t_{M}^{e}, t_{M}^{o}, t_{E}^{b}$ and $t_{E}^{e}$ as follows.

$$
\begin{aligned}
& \left\{\begin{array}{l}
t_{M}^{b}=t_{w}^{a^{*}}-\frac{u_{w}-u_{h}+\gamma}{\beta+\gamma} \frac{N}{S}, \\
t_{M}^{e}=t_{w}^{a^{*}}+\frac{u_{h}-u_{w}+\beta}{\beta+\gamma} \frac{N}{S}, \\
t_{M}^{o}=t_{w}^{a^{*}}-\frac{\left(u_{w}-u_{h}+\gamma\right)\left(u_{h}-u_{w}+\beta\right)}{(\beta+\gamma)\left(\alpha+u_{h}\right)} \frac{N}{S},
\end{array}\right. \text { for the morning commute, and } \\
& \left\{\begin{array}{l}
t_{E}^{b}=t_{w}^{d^{*}}-\frac{\widehat{u}_{h}-u_{w}+\lambda}{\mu+\lambda} \frac{N}{S}, \\
t_{E}^{e}=t_{w}^{d^{*}}+\frac{u_{w}-\widehat{u}_{h}+\mu}{\mu+\lambda} \frac{N}{S},
\end{array}\right. \text { for the evening commute. }
\end{aligned}
$$

It can be noted that when the home/work-activity utilities equal zero (i.e., $u_{h}=\widehat{u}_{h}=u_{w}=0$ ), the equilibrium solutions in Equations (27), (28), (31) and (32) are reduced to those under the 
traditional trip-based bottleneck model. This implies that the trip-based bottleneck model is a special case of the proposed activity-based bottleneck model.

The following numerical example illustrates the equilibrium solution of the activity-based model. In this example, we assume constant marginal utilities for the home and work activities, as shown in Figure 4, i.e., $u_{h}=8.0, u_{w}=11.0$ and $\widehat{u}_{h}=10.0(\$ / \mathrm{h})$. We also assume that the marginal utilities of the morning and evening home activities are different, as observed in some previous empirical studies (see, e.g., Tseng and Verhoef, 2008). The values for the parameters of the model are summarized in Table 1. In this table, it is assumed that arriving early (late) in the morning and departing late (early) in the evening are equally costly. This is consistent with that made by de Palma and Lindsey (2002a,b).

Figure 5a depicts the equilibrium solution of the activity-based bottleneck model proposed in the previous section. The curves $A_{1} B_{1} C_{1}$ and $A_{2} B_{2} C_{2}$ represent the cumulative departures from home in the morning and from work in the evening, respectively. The straight lines $A_{1} C_{1}$ and $A_{2} C_{2}$ represent the associated cumulative arrivals in the morning and the evening peaks, respectively. The values of $t_{M}^{b}, t_{M}^{e}, t_{M}^{o}, t_{E}^{b}$ and $t_{E}^{e}$ can be determined by Equations (31) and (32) as follows: $t_{M}^{b}=06: 48, t_{M}^{e}=09: 18, t_{M}^{o}=08: 38, t_{E}^{b}=16: 30$ and $t_{E}^{e}=19: 00$, respectively. For comparison, Figure $5 \mathrm{~b}$ depicts the equilibrium solution for the conventional trip-based bottleneck model. It shows that the departure times from home of the first and last commuters in the morning peak are 07:06 and 09:36, respectively. These times are 18 minutes later than those indicated by the activity-based model (06:48 and 09:18). The times of evening departure from work of the first and last commuters are 16:24 and 18:54, respectively. These times are 6 minutes earlier than those indicated by the activity-based model (16:30 and 19:00). This time difference means that the trip-based bottleneck model may lead to a biased estimation of the departure patterns for the morning and evening commuting trips compared to the activity-based bottleneck model.

We now derive the average time allocation of commuters among home/work activities and travel according to Figure 5a. Let $A T T_{M}$ and $A T T_{E}$ represent the average travel times of commuters in the morning and evening peaks, respectively. Let $A D_{h, M}$ and $A D_{h, E}$ be the average durations for their home activities in the morning and evening, respectively, and let 
$A D_{w}$ be the average work duration. They can be represented as the areas of the geometric figures shown in Figure 5a, as follows.

$$
\left\{\begin{array}{l}
A T T_{M}=\operatorname{area}\left(A_{1} B_{1} C_{1} A_{1}\right) / N, \\
A T T_{E}=\operatorname{area}\left(A_{2} B_{2} C_{2} A_{2}\right) / N, \\
A D_{h, M}=\operatorname{area}\left(O_{1} A_{1} B_{1} C_{1} G_{1} O_{1}\right) / N, \\
A D_{h, E}=\operatorname{area}\left(O_{2} A_{2} C_{2} G_{2} O_{2}\right) / N, \\
A D_{w}=\operatorname{area}\left(A_{1} A_{2} B_{2} C_{2} C_{1} A_{1}\right) / N .
\end{array}\right.
$$

Assuming that the marginal utilities of home and work activities are constants, one can easily calculate the expressions in Equation (33) as shown in Table 2.

We next define the performance indices of the system, including total travel-time costs, total schedule-delay costs and total activity utilities. Let $T T C_{M}$ and $T T C_{E}$ be the total travel-time costs of commuters in the morning and evening peaks, respectively. Let $S D C_{M}$ and $S D C_{E}$ be the total schedule-delay costs in the morning and evening peaks, respectively. Let $T U_{h, M}$ and $T U_{h, E}$ be the total utilities of home activities in the morning and evening, respectively, and let $T U_{w}$ be the total utility of work activity. These can be represented as

$$
\left\{\begin{array}{l}
T T C_{M}=\alpha \times \operatorname{area}\left(A_{1} B_{1} C_{1} A_{1}\right), \\
T T C_{E}=\alpha \times \operatorname{area}\left(A_{2} B_{2} C_{2} A_{2}\right), \\
S D C_{M}=\beta \times \operatorname{area}\left(A_{1} E_{1} F_{1} A_{1}\right)+\gamma \times \operatorname{area}\left(C_{1} E_{1} D_{1} C_{1}\right), \\
S D C_{E}=\mu \times \operatorname{area}\left(A_{2} B_{2} F_{2} A_{2}\right)+\lambda \times \operatorname{area}\left(C_{2} B_{2} D_{2} C_{2}\right), \\
T U_{h, M}=u_{h} \times \operatorname{area}\left(O_{1} A_{1} B_{1} C_{1} G_{1} O_{1}\right), \\
T U_{h, E}=\widehat{u}_{h} \times \operatorname{area}\left(O_{2} A_{2} C_{2} G_{2} O_{2}\right), \\
T U_{w}=u_{w} \times \operatorname{area}\left(A_{1} A_{2} B_{2} C_{2} C_{1} A_{1}\right) .
\end{array}\right.
$$

The detailed expressions for the system performance indices with constant marginal-activity utility are given in Table 3. It can be noted that in Tables 2 and 3, when the utilities of the home/work activities are zero, the expressions for the time-allocation and the system-performance indices in the activity-based bottleneck model are the same as those in the traditional trip-based bottleneck model. Again, this means that the trip-based bottleneck model is a special case of the activity-based bottleneck model.

In addition, it can be observed in Figures $5 \mathrm{a}$ and $\mathrm{b}$ that the areas of $A_{3} B_{3} C_{3} A_{3}$ and $A_{4} B_{4} C_{4} A_{4}$ 
are greater than those of $A_{1} B_{1} C_{1} A_{1}$ and $A_{2} B_{2} C_{2} A_{2}$, respectively. This situation indicates that the total travel times (or total queuing times) during the morning and evening commutes in the trip-based bottleneck model are higher than those in the activity-based bottleneck model. The relative increases in the total travel times (or total queuing times) during the morning and evening commutes can be respectively defined by the following two equations.

$$
\begin{aligned}
& \frac{\operatorname{area}\left(A_{3} B_{3} C_{3} A_{3}\right)-\operatorname{area}\left(A_{1} B_{1} C_{1} A_{1}\right)}{\operatorname{area}\left(A_{1} B_{1} C_{1} A_{1}\right)} \times 100 \%=\frac{\beta \gamma u_{h}+\alpha\left(u_{h}-u_{w}\right)\left(u_{h}-u_{w}+\beta-\gamma\right)}{\alpha\left(u_{h}-u_{w}+\beta\right)\left(u_{w}-u_{h}+\gamma\right)} \times 100 \%, \\
& \frac{\operatorname{area}\left(A_{4} B_{4} C_{4} A_{4}\right)-\operatorname{area}\left(A_{2} B_{2} C_{2} A_{2}\right)}{\operatorname{area}\left(A_{2} B_{2} C_{2} A_{2}\right)} \times 100 \%=\frac{\mu \lambda \widehat{u}_{h}+\alpha\left(\widehat{u}_{h}-u_{w}\right)\left(\widehat{u}_{h}-u_{w}+\lambda-\mu\right)}{\alpha\left(\hat{u}_{h}-u_{w}+\lambda\right)\left(u_{w}-\widehat{u}_{h}+\mu\right)} \times 100 \% .
\end{aligned}
$$

In view of the above discussion, we make the following proposition.

Proposition 3. Compared with the activity-based bottleneck model, the trip-based bottleneck model overestimates the total travel times (or total queuing times) during the morning and evening commutes by those given in Equations (35) and (36), respectively.

A commuter's work-activity utility may be positively correlated with his/her wage level, which usually varies with socio-economic conditions and level of urban development. The following proposition further reveals the effects of the marginal work-activity utility on the total queuing-delay costs in the morning and evening peaks.

Proposition 4. For a constant marginal-activity utility, if $-\beta<u_{h}-u_{w}<\gamma$ and $-\lambda<\widehat{u}_{h}-u_{w}<\mu$ hold, we then have

(i) The total travel-time costs (or queuing-delay costs) in the morning and evening peaks (i.e., $T T C_{M}$ and $T T C_{E}$ in Table 3) are concave with regard to the marginal work-activity utility;

(ii) Assuming that $\lambda=\beta$ and $\mu=\gamma$, there is a critical value $\bar{u}_{w}$ of the marginal work-activity utility such that when $u_{w} \leq \bar{u}_{w}\left(\right.$ or $u_{w} \geq \bar{u}_{w}$ ), the total queuing time at the bottleneck in the morning peak is longer (or shorter) than that in the evening peak.

Proof. (i) The first-order and second-order partial derivatives of $T T C_{M}$ regarding the marginal utility $u_{w}$ of work activity are 


$$
\begin{aligned}
& \frac{\partial T T C_{M}}{\partial u_{w}}=\frac{\alpha N^{2}}{2 S} \frac{2 u_{h}-2 u_{w}+\beta-\gamma}{(\beta+\gamma)\left(\alpha+u_{h}\right)}, \text { and } \\
& \frac{\partial T T C_{M}^{2}}{\partial u_{w}^{2}}=-\frac{\alpha N^{2}}{S(\beta+\gamma)\left(\alpha+u_{h}\right)}<0 .
\end{aligned}
$$

Accordingly, $T T C_{M}$ is concave with regard to $u_{w}$. In addition, for a given value of $u_{h}$, $T T C_{M}$ achieves the maximum at $u_{w}^{*}=u_{h}+\frac{1}{2}(\beta-\gamma)$ in terms of Equation (37).

Similarly, it can be easily proved that the total travel-time cost $T T C_{E}$ in the evening peak is also concave with regard to $u_{w}$, and that $T T C_{E}$ reaches the maximum at $u_{w}^{*}=\widehat{u}_{h}+\frac{1}{2}(\lambda-\mu)$.

(ii) One only needs to prove that the following equation has one unique positive root.

$$
T_{T} C_{M}=T T C_{E} \text {. }
$$

From Table 3, Equation (39) can be expressed as

$$
\frac{\left(u_{h}-u_{w}+\beta\right)\left(u_{w}-u_{h}+\gamma\right)}{(\beta+\gamma)\left(\alpha+u_{h}\right)}=\frac{\left(\widehat{u}_{h}-u_{w}+\lambda\right)\left(u_{w}-\widehat{u}_{h}+\mu\right)}{(\mu+\lambda)\left(\alpha+\widehat{u}_{h}\right)} .
$$

Equation (40) can further be written as

$$
a_{2} u_{w}^{2}+a_{1} u_{w}+a_{0}=0
$$

where

$$
\left\{\begin{array}{l}
a_{2}=\widehat{u}_{h}-u_{h}, \\
a_{1}=\left(\alpha+u_{h}\right)\left(2 \widehat{u}_{h}+\lambda-\mu\right)-\left(\alpha+\widehat{u}_{h}\right)\left(2 u_{h}+\beta-\gamma\right), \\
a_{0}=\left(\alpha+\widehat{u}_{h}\right)\left(u_{h}^{2}+u_{h}(\beta-\gamma)-\beta \gamma\right)-\left(\alpha+u_{h}\right)\left(\widehat{u}_{h}^{2}+\widehat{u}_{h}(\lambda-\mu)-\lambda \mu\right) .
\end{array}\right.
$$

Let $u_{w, 1}$ and $u_{w, 2}$ be two roots of Equation (41). As $\lambda=\beta$ and $\mu=\gamma$, we have

$$
u_{w, 1} \cdot u_{w, 2}=-\left(\alpha u_{h}+\alpha \widehat{u}_{h}+u_{h} \widehat{u}_{h}+\alpha(\beta-\gamma)+\beta \gamma\right)<0 .
$$

This implies that Equation (39) has exactly one positive root, which is given as

$$
\bar{u}_{w}=\frac{-a_{1}+\sqrt{a_{1}^{2}-4 a_{2} a_{0}}}{2 a_{2}} .
$$

It can be further shown that as $u_{w} \leq \bar{u}_{w}$, then $T T C_{M} \geq T T C_{E}$ holds, and as $u_{w} \geq \bar{u}_{w}$, then 
$T T C_{M} \leq T T C_{E}$ holds. This completes the proof of the proposition.

Proposition 4 shows that given the values of all other parameters of the model, for a city with a high marginal work-activity utility (or high average wage level), the total queuing time at the bottleneck during the evening commute is longer than that during the morning commute. Conversely, for a city with a low marginal work-activity utility (or low average wage level), the total queuing time at the bottleneck during the morning commute is longer than that during the evening commute.

\section{Linear marginal-activity utility}

In the previous section, the properties of the day-long activity-travel scheduling model (14) have been discussed, particularly for the special case with a constant marginal-activity utility. In this section, we look at another important case in which the marginal utility of activity is a linear function of the clock time. Following Jenelius et al. (2011) and Jenelius (2012), we assume that the marginal utilities of morning and evening home activities, $u_{h}(\cdot)$ and $\hat{u}_{h}(\cdot)$ are, respectively, a decreasing and an increasing function of time, and that the marginal utility of work activity $u_{w}(\cdot)$ initially increases and then decreases with regard to time. The linear marginal utility functions of the home and work activities are mathematically specified as follows:

$$
\begin{aligned}
& u_{h}(t)=g_{0}+g_{1} t, \quad g_{1}<0, \\
& u_{w}\left(t-\xi t_{w}^{a}\right)= \begin{cases}h_{0}+h_{1}\left(t-\xi t_{w}^{a}\right), & t-\xi t_{w}^{a} \leq \underline{t}, h_{1}>0, \\
k_{0}+k_{1}\left(t-\xi t_{w}^{a}\right), & t-\xi t_{w}^{a} \geq \bar{t}, k_{1}<0,\end{cases} \\
& \hat{u}_{h}(t)=l_{0}+l_{1} t, l_{1}>0,
\end{aligned}
$$

where $\underline{t}$ represents the time at which the marginal utility of the work activity stops growing in the morning (i.e., the end of the warm-up period), and $\bar{t}(\bar{t} \geq \underline{t})$ represents the time at which the marginal utility of the work activity in the evening starts to drop (i.e., the start of the cool-down period). When $t \leq \underline{t}+\xi t_{w}^{a}\left(t \geq \bar{t}+\xi t_{w}^{a}\right)$, the marginal utility of the work activity is an increasing (or a decreasing) function of time $t$.

For illustration purpose, Figure 6 shows an example of the linear marginal utility functions for 
home and work activities. In this figure, when the work start time falls in the interval $\left[\underline{t}+\xi t_{w}^{a}, \bar{t}+\xi t_{w}^{a}\right]$, the marginal utility of the work activity is a constant of $h_{0}+h_{1} \underline{t}$ or $k_{0}+k_{1} \bar{t}$. According to Proposition 2, if $\xi \neq 0$, the commuters' morning and evening departure-time choices are interdependent.

With the linear marginal-activity utility specifications as described above, the total utilities of the morning and evening home activities can be respectively expressed as follows:

$$
\begin{aligned}
& \int_{0}^{t_{h}^{d}} u_{h}(t) d t=\int_{0}^{t_{h}^{d}}\left(g_{0}+g_{1} t\right) d t=g_{0} t_{h}^{d}+0.5 g_{1} \times\left(t_{h}^{d}\right)^{2}, \\
& \int_{t_{h}^{a}}^{24} \hat{u}_{h}(t) d t=\int_{t_{h}^{a}}^{24}\left(l_{0}+l_{1} t\right) d t=l_{0}\left(24-t_{h}^{a}\right)+0.5 l_{1}\left(24^{2}-\left(t_{h}^{a}\right)^{2}\right) .
\end{aligned}
$$

We now calculate the total utility of the work activity during the day, i.e., $\int_{t_{w}^{a}}^{t_{w}^{d}} u_{w}\left(t-\xi t_{w}^{a}\right) d t$. It can be seen in Figure 6 that the end of the warm-up period (i.e., $\left.\underline{t}+\xi t_{w}^{a}\right)$ and the start of the cool-down period (i.e., $\bar{t}+\xi t_{w}^{a}$ ) divide each whole day into three periods: $\left[0, \underline{t}+\xi t_{w}^{a}\right]$, $\left[\underline{t}+\xi t_{w}^{a}, \bar{t}+\xi t_{w}^{a}\right]$ and $\left[\bar{t}+\xi t_{w}^{a}, 24\right]$. The lower limit $t_{w}^{a}$ of the integral $\int_{t_{w}^{a}}^{t_{w}^{d}} u_{w}\left(t-\xi t_{w}^{a}\right) d t$ (i.e., the arrival time at the workplace in the morning) and the upper limit $t_{w}^{d}$ of the integral (i.e., the departure time from work in the evening) may be located in any one of these three periods, subject to the constraint of $t_{w}^{a} \leq t_{w}^{d}$. There are a total of six possible cases for locating the relationship between $t_{w}^{a}$ and $t_{w}^{d}$, as shown in Table 4. The corresponding work-activity utility $\int_{t_{w}^{a}}^{t_{w}^{d}} u_{w}\left(t-\xi t_{w}^{a}\right) d t$ for these six cases can, respectively, be given as follows:

(i) When $t_{w}^{a}$ and $t_{w}^{d} \in\left[0, \underline{t}+\xi t_{w}^{a}\right]$,

$$
\int_{t_{w}^{a}}^{t_{w}^{d}} u_{w}\left(t-\xi t_{w}^{a}\right) d t=\int_{t_{w}^{a}}^{t_{w}^{d}}\left(h_{0}+h_{1}\left(t-\xi t_{w}^{a}\right)\right) d t=\left(h_{0}-h_{1} \xi t_{w}^{a}\right)\left(t_{w}^{d}-t_{w}^{a}\right)+0.5 h_{1}\left(\left(t_{w}^{d}\right)^{2}-\left(t_{w}^{a}\right)^{2}\right) .
$$

(ii) When $t_{w}^{a} \in\left[0, \underline{t}+\xi t_{w}^{a}\right]$ and $t_{w}^{d} \in\left[\underline{t}+\xi t_{w}^{a}, \bar{t}+\xi t_{w}^{a}\right]$,

$$
\int_{t_{w}^{a}}^{t_{w}^{d}} u_{w}\left(t-\xi t_{w}^{a}\right) d t=\int_{t_{w}^{a}}^{\underline{t}+\xi t_{w}^{a}}\left(h_{0}+h_{1}\left(t-\xi t_{w}^{a}\right)\right) d t+\left(h_{0}+h_{1} \underline{t}\right) \int_{\underline{t}+\xi t_{w}^{d}}^{t_{w}^{d}} d t
$$




$$
=\left(h_{0}-h_{1} \xi t_{w}^{a}\right)\left(\underline{t}+\xi t_{w}^{a}-t_{w}^{a}\right)+0.5 h_{1}\left(\left(\underline{t}+\xi t_{w}^{a}\right)^{2}-\left(t_{w}^{a}\right)^{2}\right)+\left(t_{w}^{d}-\underline{t}-\xi t_{w}^{a}\right)\left(h_{0}+h_{1} \underline{t}\right) .
$$

(iii) When $t_{w}^{a} \in\left[0, \underline{t}+\xi t_{w}^{a}\right]$ and $t_{w}^{d} \in\left[\bar{t}+\xi t_{w}^{a}, 24\right]$,

$$
\begin{aligned}
& \int_{t_{w}^{a}}^{t_{w}^{d}} u_{w}\left(t-\xi t_{w}^{a}\right) d t=\int_{t_{w}^{a}}^{\underline{t}+\xi t_{w}^{a}}\left(h_{0}+h_{1}\left(t-\xi t_{w}^{a}\right)\right) d t+\left(h_{0}+h_{1} \underline{t}\right) \int_{\underline{t}+\xi t_{w}^{a}}^{\bar{t} \xi \xi t_{w}^{a}} d t+\int_{\bar{t}+\xi t_{w}^{a}}^{t_{w}^{d}}\left(k_{0}+k_{1}\left(t-\xi t_{w}^{a}\right)\right) d t \\
& =\left(h_{0}-h_{1} \xi t_{w}^{a}\right)\left(\underline{t}+\xi t_{w}^{a}-t_{w}^{a}\right)+0.5 h_{1}\left(\left(\underline{t}+\xi t_{w}^{a}\right)^{2}-\left(t_{w}^{a}\right)^{2}\right)+(\bar{t}-\underline{t})\left(h_{0}+h_{1} \underline{t}\right) \\
& +\left(k_{0}-k_{1} \xi t_{w}^{a}\right)\left(t_{w}^{d}-\bar{t}-\xi t_{w}^{a}\right)+0.5 k_{1}\left(\left(t_{w}^{d}\right)^{2}-\left(\bar{t}+\xi t_{w}^{a}\right)^{2}\right) .
\end{aligned}
$$

(iv) When $t_{w}^{a}$ and $t_{w}^{d} \in\left[\underline{t}+\xi t_{w}^{a}, \bar{t}+\xi t_{w}^{a}\right]$,

$$
\int_{t_{w}^{a}}^{t_{w}^{d}} u_{w}\left(t-\xi t_{w}^{a}\right) d t=\left(t_{w}^{d}-t_{w}^{a}\right)\left(h_{0}+h_{1} \underline{t}\right) .
$$

(v) When $t_{w}^{a} \in\left[\underline{t}+\xi t_{w}^{a}, \bar{t}+\xi t_{w}^{a}\right]$ and $t_{w}^{d} \in\left[\bar{t}+\xi t_{w}^{a}, 24\right]$,

$$
\begin{aligned}
& \int_{t_{w}^{a}}^{t_{w}^{d}} u_{w}\left(t-\xi t_{w}^{a}\right) d t=\left(h_{0}+h_{1} \underline{t}\right) \int_{t_{w}^{a}}^{\bar{t}+\xi t_{w}^{a}} d t+\int_{\bar{t}+\xi t_{w}^{a}}^{t_{w}^{d}}\left(k_{0}+k_{1}\left(t-\xi t_{w}^{a}\right)\right) d t \\
& =\left(\bar{t}+\xi t_{w}^{a}-t_{w}^{a}\right)\left(h_{0}+h_{1} \underline{t}\right)+\left(k_{0}-k_{1} \xi t_{w}^{a}\right)\left(t_{w}^{d}-\bar{t}-\xi t_{w}^{a}\right)+0.5 k_{1}\left(\left(t_{w}^{d}\right)^{2}-\left(\bar{t}+\xi t_{w}^{a}\right)^{2}\right) .
\end{aligned}
$$

(vi) When $t_{w}^{a}$ and $t_{w}^{d} \in\left[\bar{t}+\xi t_{w}^{a}, 24\right]$,

$$
\int_{t_{w}^{a}}^{t_{w}^{d}} u_{w}\left(t-\xi t_{w}^{a}\right) d t=\int_{t_{w}^{a}}^{t_{w}^{d}}\left(k_{0}+k_{1}\left(t-\xi t_{w}^{a}\right)\right) d t=\left(k_{0}-k_{1} \xi t_{w}^{a}\right)\left(t_{w}^{d}-t_{w}^{a}\right)+0.5 k_{1}\left(\left(t_{w}^{d}\right)^{2}-\left(t_{w}^{a}\right)^{2}\right) .
$$

It should be pointed out that when $\underline{t}$ and $\bar{t}$ are equal, the interval $\left[\underline{t}+\xi t_{w}^{a}, \bar{t}+\xi t_{w}^{a}\right]$ becomes a time point, and cases (ii), (iv) and (v) thus immediately disappear. In other words, there are only three cases, i.e., (i), (iii) and (vi) for which $\underline{t}=\bar{t}$. We will later illustrate this situation with an example.

In light of the above discussion, if we are given a departure-time pair $\left(t_{h}^{d}, t_{w}^{d}\right)$, we can then directly calculate the total utility of the morning home activity according to Equation (48). However, to determine the total utility of the evening home activity by Equation (49) and of the work activity by Equations (50)-(55), we must first determine the queuing delays $T_{E}\left(t_{w}^{d}\right)$ 
and $T_{M}\left(t_{h}^{d}\right)$, and then $t_{h}^{a}$ and $t_{w}^{a}$ in terms of $t_{h}^{a}=t_{w}^{d}+T_{E}\left(t_{w}^{d}\right)$ and $t_{w}^{a}=t_{h}^{d}+T_{M}\left(t_{h}^{d}\right)$, respectively. For the constant marginal-activity utility case, one can easily determine the closed-form expressions of $T_{M}\left(t_{h}^{d}\right)$ and $T_{E}\left(t_{w}^{d}\right)$ and the constant equilibrium departure rates $r_{M}\left(t_{h}^{d}\right)$ and $r_{E}\left(t_{w}^{d}\right)$. However, for the linear marginal-activity utility case, we cannot obtain the closed-form solutions of these quantities, and thus must turn to a numerical approach. In the following, a heuristic solution algorithm based on the method of successive averages (MSA) is presented to solve the activity-travel scheduling model (14) with a linear marginal-activity utility function.

Step 0. Choose an initial departure flow pattern $q^{(0)}\left(t_{h}^{d}, t_{w}^{d}\right)$ for any departure-time pair $\left(t_{h}^{d}\right.$, $\left.t_{w}^{d}\right)$ and set $i=1$.

Step 1. Calculate the net utility $U^{(i)}\left(t_{h}^{d}, t_{w}^{d}\right)$ according to Equation (13) and determine the "best" departure-time pair $\left(t_{h}^{d}, t_{w}^{d}\right)^{*}$ that maximizes the net utility over all times of the day.

Step 2. Assign all the commuters to the "best" pair $\left(t_{h}^{d}, t_{w}^{d}\right)^{*}$ and obtain the auxiliary departure flow pattern $\hat{q}^{(i)}\left(t_{h}^{d}, t_{w}^{d}\right)$.

Step 3. Make use of the MSA to update the departure flow pattern:

$$
q^{(i+1)}\left(t_{h}^{d}, t_{w}^{d}\right)=q^{(i)}\left(t_{h}^{d}, t_{w}^{d}\right)+\frac{1}{i+1}\left(\hat{q}^{(i)}\left(t_{h}^{d}, t_{w}^{d}\right)-q^{(i)}\left(t_{h}^{d}, t_{w}^{d}\right)\right) .
$$

Step 4. If $\frac{\sqrt{\sum_{t_{h}^{d}} \sum_{t_{w}^{d}}\left(\hat{q}^{(i)}\left(t_{h}^{d}, t_{w}^{d}\right)-q^{(i)}\left(t_{h}^{d}, t_{w}^{d}\right)\right)^{2}}}{\sum_{t_{h}^{d}} \sum_{t_{w}^{d}} q^{(i)}\left(t_{h}^{d}, t_{w}^{d}\right)}<\varepsilon$ (where $\varepsilon$ is a pre-specified precision or tolerance), then stop. Otherwise, set $i=i+1$ and go to Step 1 .

In Step 0, the initial departure flow pattern $q^{(0)}\left(t_{h}^{d}, t_{w}^{d}\right)$ can be set to zero, which signifies an empty state at the beginning of the day. Once the optimal departure-flow pattern is obtained, one can then determine the morning home-to-work departure rate $r_{M}\left(t_{h}^{d}\right)$ and the evening work-to-home departure rate $r_{E}\left(t_{w}^{d}\right)$ by Equations (16) and (17), respectively. The morning and evening queue lengths and queue times can be determined by Equations (3)-(8), the schedule delays by Equations (9) and (10), the activity utility by Equation (12) and finally the 
total net utility of each commuter for the whole day by Equation (13). The MSA-based solution algorithm has also been adopted in previous related studies, such as those by Zhang et al. (2005) and Huang and Lam (2005).

\section{Numerical studies}

In this section, two test scenarios are used to illustrate the properties of the proposed model and the contributions of this paper. The first scenario, which is concerned with a constant marginal-activity utility, is applied to ascertain the effects of the work-activity utility on the morning/evening queuing-delay costs and to compare the solutions of the activity-based and trip-based models. The second scenario, which takes account of a linear marginal-activity utility function, is used to investigate the effects of flexibility in work hours on the commuters' time allocations and the system performance.

\subsection{Scenario 1: Constant marginal-activity utility}

The model input parameters for Scenario 1 are the same as those shown in Table 1. We first explore the effects of the work-activity utility on morning/evening queuing-delay costs. Figure 7 shows the change in the total queuing-delay costs, $T T C_{M}$ and $T T C_{E}$, in the morning and evening peaks in relation to the marginal utility of the work activity (i.e., $u_{w}$ ) when $u_{h}$ and $\hat{u}_{h}$ are fixed as $\$ 8.0$ and $\$ 10.0$ per hour, respectively. It can be seen that both the morning and evening queuing-delay cost curves are concave. As $u_{w}$ increases, the total queuing-delay costs $\left(T T C_{M}\right.$ and $\left.T T C_{E}\right)$ in the morning and evening peaks first increase and then decrease, achieving their maxima at points X1 and X2, with $u_{w}=1.5$ and $3.5(\$ / \mathrm{h})$, respectively. This scenario leads to total queuing-delay costs of $\$ 21,701$ and $\$ 19,531$ in the morning and evening peaks, respectively. These two queuing-delay cost curves intersect at point X3, with a marginal-utility level of $\bar{u}_{w}=6.2(\$ / \mathrm{h})$ in terms of Equation (44), which gives a total queuing-delay cost of $\$ 18,607$. As $u_{w} \leq \bar{u}_{w}$, the total queuing time in the morning peak is longer than that in the evening peak.

We now compare the equilibrium solutions under different modeling approaches (i.e., the activity-based and trip-based models). Figure 8 shows the resultant queue lengths over the 
times of a day under the activity- and trip-based approaches. It can be seen that the queue-length curves for both approaches take triangular forms. Specifically, for the activity-based approach, the queue length in the morning increases linearly from zero at 06:48 to the maximum (733 vehicles) at $t_{M}^{o}$ (i.e., 08:38). The queue length then decreases linearly to zero at 09:18. During the evening commute, the queue length increases linearly from zero at $16: 30$ to the maximum (1,000 vehicles) at the preferred departure time of 17:00, and then decreases linearly to zero at 19:00. It can also be observed that under the activity-based approach, the area of the triangle for the queue length in the morning is smaller than that in the evening. However, under the trip-based approach, the areas of the triangles associated with the morning and evening queuing are the same, implying that for the trip-based approach, the queue pattern in the morning is a mirror image of the evening pattern.

Tables 5 and 6 further indicate the equilibrium time allocation of commuters and the performance of the system under the activity-based and trip-based approaches. Table 5 shows that compared to the activity-based approach, the trip-based approach overestimates the average travel times in the morning and evening peaks by 0.39 and 0.32 hours, respectively, and overestimates the average duration for evening home activity by 0.10 hour. It also underestimates the average duration of morning home activity by 0.09 hour and the average work duration by 0.72 hour. Table 6 shows that the trip-based approach overestimates the total travel-time costs in the morning and evening peaks by $\$ 19,333$ and $\$ 16,000$, respectively, and overestimates the total utility of evening home activity by $\$ 5,000$. However, it underestimates the total utilities of the morning home activity and the work activity by $\$ 3,467$ and $\$ 39,600$, respectively. As a result, the trip-based approach underestimates the total net utility of the system (with a total of 5,000 commuters) by $\$ 72,150$ per day $(\$ 994,850$ per day compared to $\$ 1,067,000$ per day for the activity-based approach). Thus, the average net utility of each commuter is underestimated by $\$ 14.43$ per day ( $\$ 198.97$ per day compared to $\$ 213.40)$. These results indicate that the trip-based bottleneck model may lead to a biased estimation of the commuters' time-use decisions and of the system's performance.

\subsection{Scenario 2: Linear marginal-activity utility}

In this section, we illustrate the case with the linear marginal-activity utility. The linear marginal-activity utility functions for home and work activities are shown in Figure 9. It is 
assumed that both $\underline{t}$ and $\bar{t}$ are equal to 12:00, implying that three cases exist for the location between $t_{w}^{a}$ and $t_{w}^{d}$, i.e., (i), (iii) and (vi), as shown in Section 4. All other model input parameters are identical with those given in Table 1. As there is no closed-form solution for this case, we have to adopt the heuristic solution algorithm as presented in Section 4. The proposed solution algorithm has been coded in the $\mathrm{C}$ programming language and run on a personal computer with an Intel Pentium 1.4-GHz CPU and 1 GB of RAM.

To look at the performance of the proposed heuristic solution algorithm, we apply the algorithm to the constant marginal-activity utility case examined in Section 5.1. Figure 10 reports the numerical solutions of departure flow rate with different levels of precision (0.0001 and 0.00001), along with the analytical solution calculated directly from Equations (27) and (28). It can be seen that as the precision increases, the difference between the numerical and analytical solutions diminishes. However, the CPU time required for the numerical computation increases. Specifically, the CPU times needed with the precision levels of 0.0001 and 0.00001 are 617 and 5,700 seconds, respectively. In the following experiments, the convergence precision is set as 0.00001 .

Figure 11 displays the departure flow patterns during the morning and evening peaks and the associated net utility for three different values of the flexibility parameter $\xi$ (i.e., $\xi=0.0$, 0.3 , and 1.0). These three values stand for the rigid, partially flexible, and totally flexible work-hour schemes, respectively. It can be noted that for a given value of $\xi$, the net utilities of commuters are exactly equal and are maximum for all chosen departure times, implying that the departure-time choice equilibrium is really achieved. Among these three schemes, the equilibrium net utility with $\xi=0.3$ is largest ( $\$ 192.69$ per day), that with $\xi=1.0$ is smallest ( $\$ 167.05$ per day) and that with $\xi=0.0$ is in between ( $\$ 188.72$ per day).

It can also be noted that the departure rates during the morning and evening peaks change linearly, unlike the departure rates in the constant marginal-activity utility case, which are constant (see Equations 27 and 28 or Figure 10). In particular, within the departure periods, the morning and evening departure rates decrease linearly for a small $\xi$-value (e.g., $\xi=0.0$ and 0.3 ), but increase linearly for the case of $\xi=1.0$. In addition, compared to the rigid scheme of $\xi=0.0$, the partially flexible scheme of $\xi=0.3$ and the totally flexible scheme of 
$\xi=1.0$ lead commuters to postpone their off-work time in the evening. However, the former (i.e., $\xi=0.3)$ results in a more centralized morning departure period, and the latter (i.e., $\xi=$ 1.0) causes a more decentralized departure period. As a result, the total queuing delay with $\xi=0.3$ during the morning peak is highest, whereas the delays with $\xi=1.0$ during the morning and evening peaks are always lowest, as shown in Figure 12. In addition, Figure 12 shows that the changes in queue length are no longer linear over the times of the day as they are in the constant marginal-activity utility case (see Figure 8). According to Equations (3) and (4) and the linear departure-rate patterns shown in Figure 11, the change curves in the queue lengths seem to be quadratic. This finding deserves an in-depth investigation in a further study.

Table 7 further shows the effects of the flexibility parameter $\xi$ on commuters' patterns of time allocation and average net utility during a day. This parameter shows that when $\xi$ does not exceed 0.7, the commuter's average work duration always increases, whereas the average queuing duration and the average home duration decrease. As a result, the average net utility for commuters first increases and then decreases, with the maximum occurring at the flexibility level of $\xi=0.3$. When $\xi$ is larger than 0.7 (i.e., $\xi=0.8,0.9$ or 1.0 ), the effect on the commuters' allocations of time to activities and travel during the day is modest. These observations show that introducing a flexible work-hour scheme can change workers' activity schedules and time-use decisions. The workers' productivity could be impaired when coworkers are absent during a large portion of the workday. Consequently, when implementing a flexible work-hour scheme, the degree of flexibility should be carefully designed such that the workers can use their time during a day more efficiently.

Finally, we investigate the effects of the flexibility parameter $\xi$ on the performance of the system, as shown in Figure 13. It can be seen that as $\xi$ increases, the total activity utility of the system first increases and then decreases. The maximum total activity utility comes at point $\mathrm{Y} 1$, with a flexibility of $\xi=0.2$ and a total activity utility of $\$ 1.055$ million per day. The total schedule-delay cost first decreases and then increases, reaching the minimum at point $\mathrm{Y} 3$, which is associated with a flexibility of $\xi=0.4$ and a total schedule-delay cost of $\$ 54,720$ per day. However, the total queuing-delay cost always decreases with the increase of flexibility. As a result of the tradeoff among the utility and cost components, the total user net 
utility of the system achieves its maximum at point Y2, with a flexibility of $\xi=0.3$ and a total net utility of $\$ 0.965$ million per day. This result shows that either a rigid or a totally flexible work-hour scheme could cause low work efficiency and low user net utility. There is an optimal partially flexible work-hour scheme that yields the maximum total user net utility.

\section{Conclusion and areas for further study}

In this paper, we extend the conventional bottleneck model to address the activity and travel scheduling problems of workers during the course of a day by using an activity-based approach. A typical activity chain for a worker, i.e., the "home-work-home" tour, is considered. Given the definition of total net utility for the typical tour, we propose an activity-travel scheduling model that maximizes the total net utility for this tour within a day. The properties of the equilibrium model are analytically explored and compared with those of the standard bottleneck model, particularly for a special case with constant marginal-activity utility. For the case with linear marginal-activity utility, we develop a heuristic solution algorithm to find its equilibrium solution. The effects of flexibility in work-hour schemes on the scheduling of morning and evening commuting tours are also explored.

This paper offers some new insights and important findings. First, we present a sufficient and necessary condition that a queue exists at the bottleneck during the morning and evening commutes (see Proposition 1 and Corollary 1), along with a condition of interdependence between the morning and evening departure-time decisions (see Proposition 2). Second, it is shown that the conventional bottleneck model is a special case of the activity-based bottleneck model proposed here. The trip-based model might lead to a biased estimation of the commuters' time-allocation decisions and of the system performance (see Proposition 3). Third, the bottleneck queuing delays in the morning and evening peaks are shown to be concave with regard to the marginal utility of work activity. A sufficient condition for identifying whether the morning or evening average queuing delays will be heavier is also proposed (see Proposition 4). Fourth, the implementation of a flexible work-hour scheme is shown able to change the commuters' activity schedules and their time-use decisions. An appropriate flexibility in work hours can be more productive than either a rigid or a totally flexible scheme in terms of total net utility of the system. The proposed model can serve as a useful tool for modeling the commuters' daily time allocations among activities and trips, and 
their departure-time choices.

Although the model proposed in this paper provides useful insights for practical policy analysis and evaluation, some important extensions should be made in future studies. These extensions include the following.

(1) The proposed model assumes that all commuters are homogenous in terms of their work arrival/departure-time preferences, values of their time and costs of schedule delays. However, other studies have shown that there are big differences in the choice behavior of heterogeneous commuters. The assumption of commuter homogeneity should be relaxed to incorporate the heterogeneity of commuters in a further study.

(2) This paper mainly focuses on the travel and activity scheduling problems of commuters over the times of an individual day. Some other effective measures for bottleneck congestion relief are not addressed here, such as congestion pricing or tradable credit schemes (Yang and Wang, 2011; Xiao et al., 2013; Nie and Yin, 2013). It should be worthwhile to extend the proposed model to investigate these issues.

(3) Parking issues in downtown areas are not considered in this paper. It has been shown that parking availability can influence the activity and trip schedules of commuters, thereby affecting the dynamics of traffic congestion at the bottleneck (Zhang et al., 2008, 2011; Arnott and Rowse, 2009; Qian et al., 2012; Yang et al., 2013). It is thus important to explicitly incorporate the effects of parking policies on commuters' activity and travel scheduling into the bottleneck model in a future study.

(4) Only the auto mode is examined in this paper, and thus the interaction between auto and other transit modes is not considered. In reality, the accessibilities and service levels of various competitive modes (e.g., auto, bus and metro) do affect commuters' activity plans and time allocations. It should therefore be meaningful to incorporate other travel mode options and the interactions between mixed forms of traffic into the activity-travel scheduling model.

(5) In this paper, a constant and a linear marginal-activity utility function are analyzed. A step marginal-activity utility function may be more sensible for practical applications, and this approach deserves further study. In addition, there is a need to empirically calibrate the utility 
functions of various activities through field surveys.

\section{Acknowledgements}

The authors would like to thank two anonymous referees for their helpful comments and constructive suggestions on an earlier draft of the paper. The work described in this paper was jointly supported by grants from the National Natural Science Foundation of China (71222107, 71171013), the National Basic Research Program of China (973 Program) (2012CB725400), and the Research Grants Council of the Hong Kong Special Administrative Region, China (PolyU5181/13E, HKU7175/12E).

\section{References}

Arnott, R., de Palma, A., Lindsey, R., 1988. Schedule delay and departure time decisions with heterogeneous commuters. Transportation Research Record 1197, 56-67.

Arnott, R., de Palma, A., Lindsey, R., 1990. Economics of a bottleneck. Journal of Urban Economics 27 (1), 111-130.

Arnott, R., de Palma, A., Lindsey, R., 1992. Route choice with heterogeneous drivers and group-specific congestion costs. Regional Science and Urban Economics 22 (1), 71-102.

Arnott, R., de Palma, A., Lindsey, R., 1993. A structural model of peak-period congestion: A traffic bottleneck with elastic demand. American Economic Review 83 (1), 161-179.

Arnott, R., de Palma, A., Lindsey, R., 1998. Recent developments in the bottleneck model. In: Button, K.J., Verhoef, E.T. (eds.), Road Pricing, Traffic Congestion and the Environment: Issues of Efficiency and Social Feasibility, pp. 79-110. Edward Elgar, Aldershot.

Arnott, R., Kraus, M., 1995. Financing capacity in the bottleneck model. Journal of Urban Economics 38 (3), 272-290.

Arnott, R., Rowse, J., 2009. Downtown parking in auto city. Regional Science and Urban Economics 39 (1), 1-14.

Ashiru, O., Polak, J.W., Noland, R.B., 2004. Utility of schedules: Theoretical model of departure-time choice and activity-time allocation with application to individual activity schedules. Transportation Research Record 1894, 84-98.

Daganzo, C.F., 2013. System optimum and pricing for the day-long commute with distributed demand, autos and transit. Transportation Research Part B 55, 98-117. 
de Palma, A., Lindsey, R., 2002a. Comparison of morning and evening commutes in the Vickrey bottleneck model. Transportation Research Record 1807, 26-33.

de Palma, A., Lindsey, R., 2002b. Congestion pricing in the morning and evening peaks: A comparison using the bottleneck model. Proceedings of the 37th Annual Conference of the Canadian Transportation Research Forum: 2002 Transportation Visioning - 2002 and Beyond. Vancouver, pp. 179-193.

Ettema, D., Bastin, F., Polak, J.W., Ashiru, O., 2007. Modeling the joint choice of activity timing and duration. Transportation Research Part A 41 (9), 827-841.

Ettema, D., Timmermans, H.J.P., 1997. Activity-Based Approaches to Travel Analysis. Pergamon, Oxford.

Ettema, D., Timmermans, H.J.P., 2003. Modeling departure time choice in the context of activity scheduling behavior. Transportation Research Record 1831, 39-46.

Fargier, P.H., 1983. Effects of the choice of departure time on road traffic congestion: Theoretical approach. Proceedings of the 8th International Symposium on Transportation and Traffic Theory, pp. 223-263. University of Toronto Press, Toronto, Canada.

Fosgerau, M., Lindsey, R., 2013. Trip-timing decisions with traffic incidents. Regional Science and Urban Economics 43 (5), 764-782.

Fu, X., Lam, W.H.K., 2014. A network equilibrium approach for modeling activity-travel pattern scheduling problems in multi-modal transit networks with uncertainty. Transportation 41 (1), 37-55.

Gonzales, E.J., Daganzo, C.F., 2013. The evening commute with cars and transit: Duality results and user equilibrium for the combined morning and evening peaks. Transportation Research Part B 57, 286-299.

Huang, H.J., 2002. Pricing and logit-based mode choice models of a transit and highway system with elastic demand. European Journal of Operational Research 140, 562-570.

Huang, H.J., Lam, W.H.K., 2005. A stochastic model for combined activity/destination/route choice problem. Annals of Operations Research 135, 111-125.

Huang, H.J., Tian, Q., Yang, H., Gao, Z.Y., 2007. Modal split and commuting pattern on a bottleneck-constrained highway. Transportation Research Part E 43 (5), 578-590.

Huang, H.J., Yang, H., 1996. Optimal variable road-use pricing on a congested network of parallel routes with elastic demand. In: Lesort, J.B. (ed.), Transportation and Traffic Theory, pp. 479-500. Elsevier, Oxford.

Hurdle, V.F., 1981. Equilibrium flows on urban freeways. Transportation Science 15 (3), 255-293. 
Jenelius, E., 2012. The value of travel time variability with trip chains, flexible scheduling and correlated travel times. Transportation Research Part B 46 (6), 762-780.

Jenelius, E., Mattsson, L.G., Levinson, D., 2011. Traveler delay costs and value of time with trip chains, flexible activity scheduling and information. Transportation Research Part B 45 (5), 789-807.

Jones, P., Koppelman, F.S., Orfeuil, J.P., 1990. Activity analysis: State of the art and future directions. In: Jones, P. (ed.) Developments in Dynamic and Activity-Based Approaches to Travel Analysis, pp. 34-55. Avebury, Aldershot, England.

Kawakami, S., Isobe, T., 1990. Development of a one-day travel-activity scheduling model for workers. In: Jones, P. (ed.) Development in Dynamic and Activity-Based Approaches to Travel Analysis, pp.184-205. Avebury, Aldershot, England.

Kitamura, R., 1988. An evaluation of activity-based travel analysis. Transportation 15 (1), 9-34.

Lam, W.H.K., Huang, H.J., 2002. A combined activity/travel choice model for congested road networks with queues. Transportation 29 (1), 5-29.

Lam, W.H.K., Yin, Y., 2001. An activity-based time-dependent traffic assignment model. Transportation Research Part B 35 (6), 549-574.

Li, Z.C., Lam, W.H.K., Wong, S.C., Sumalee, A., 2010. An activity-based approach for scheduling multimodal transit services. Transportation 37 (5), 751-774.

Lindsey, R., 2004. Existence, uniqueness, and trip cost function properties of user equilibrium in the bottleneck model with multiple user classes. Transportation Science 38 (3), 293-314.

Lindsey, R., van den Berg, V.A.C., Verhoef, E.T., 2012. Step tolling with bottleneck queuing congestion. Journal of Urban Economics 72 (1), 46-59.

Lindsey, R., Verhoef, E.T., 2001. Traffic congestion and congestion pricing. In: Button, K.J., Hensher, D.A. (eds.), Handbook of Transport Systems and Traffic Control, pp. 77-105. Pergamon, Amsterdam.

Nie, Y., Yin, Y., 2013. Managing rush hour travel choices with tradable credit scheme. Transportation Research Part B 50, 1-19.

Qian, Z., Xiao, F., Zhang, H.M., 2012. Managing morning commute traffic with parking. Transportation Research Part B 46 (7), 894-916.

Qian, Z., Zhang, H.M., 2013. The morning commute problem with heterogeneous travellers: The case of continuously distributed parameters. Transportmetrica A 9 (2), 178-203.

Siu, B.W.Y., Lo, H.K., 2013. Punctuality-based departure time scheduling under stochastic 
bottleneck capacity: Formulation and equilibrium. Transportmetrica B 1 (3), 195-225.

Small, K.A., 1982. The scheduling of consumer activities: Work trips. American Economic Review 72 (3), 467-479.

Tabuchi, T., 1993. Bottleneck congestion and modal split. Journal of Urban Economics 34 (3), 414-431.

Timmermans, H.J.P., 2005. Progress in Activity-Based Analysis. Elsevier, Amsterdam.

Tseng, Y.Y., Verhoef, E.T., 2008. Value of time by time of day: A stated-preference study. Transportation Research Part B 42 (7-8), 607-618.

van den Berg, V.A.C., 2014. Coarse tolling with heterogeneous preferences. Transportation Research Part B 64, 1-23.

van den Berg, V.A.C., Verhoef, E.T., 2011. Congestion tolling in the bottleneck model with heterogeneous values of time. Transportation Research Part B 45 (1), 60-78.

van den Berg, V.A.C., Verhoef, E.T., 2014. Congestion pricing in a road and rail network with heterogeneous values of time and schedule delay. Transportmetrica A 10 (5), 377-400.

Vickrey, W.S., 1969. Congestion theory and transport investment. American Economic Review (Papers and Proceedings) 59 (2), 251-260.

Vickrey, W.S., 1973. Pricing, metering, and efficiently using urban transportation facilities. Highway Research Record 476, 36-48.

Wang, J.J., 1996. Timing utility of daily activities and its impact on travel. Transportation Research Part A 30, 189-206.

Xiao, F., Qian, Z., Zhang, H.M., 2011. The morning commute problem with coarse toll and nonidentical commuters. Networks and Spatial Economics 11 (2), 343-369.

Xiao, F., Qian, Z., Zhang, H.M., 2013. Managing bottleneck congestion with tradable credits. Transportation Research Part B 56, 1-14.

Xiao, F., Shen, W., Zhang, H.M., 2012. The morning commute under flat toll and tactical waiting. Transportation Research Part B 46 (10), 1346-1359.

Xiao, L.L., Huang, H.J., Liu, R.H., 2014. Congestion behavior and tolls in a bottleneck model with stochastic capacity. Transportation Science, in press.

Yang, H., Liu, W., Wang, X.L., Zhang, X.N., 2013. On the morning commute problem with bottleneck congestion and parking space constraints. Transportation Research Part B 58, 106-118.

Yang, H., Wang, X.L., 2011. Managing network mobility with tradable credits. Transportation Research Part B 45 (3), 580-594.

Zhang, X.N., Huang, H.J., Zhang, H.M., 2008. Integrated daily commuting patterns and 
optimal road tolls and parking fees in a linear city. Transportation Research Part B 42 (1), $38-56$.

Zhang, X.N., Yang, H., Huang, H.J., 2011. Improving travel efficiency by parking permits distribution and trading. Transportation Research Part B 45 (7), 1018-1034.

Zhang, X.N., Yang, H., Huang, H.J., Zhang, H.M., 2005. Integrated scheduling of daily work activities and morning-evening commutes with bottleneck congestion. Transportation Research Part A 39 (1), 41-60. 


\section{Appendix A. Proof of Proposition 1}

We only provide the proof of part (i) here. The proof of part (ii) is similar to that of part (i), and is omitted to save space, but is available from the authors on request.

(Necessity) We need to prove that a queue exists at the bottleneck, as implied in Equation (18). A contradiction method is adopted here. Suppose that Equation (18) does not hold, which implies

$$
\left\{\begin{array}{l}
u_{h}(t) \leq \xi u_{w}\left(t_{w}^{d}-\xi t\right)-(\xi-1) u_{w}(t-\xi t)-\beta, \text { for } t \in\left(t_{M}^{b}, t_{M}^{o}\right], \\
u_{h}(t) \geq \xi u_{w}\left(t_{w}^{d}-\xi t\right)-(\xi-1) u_{w}(t-\xi t)+\gamma, \text { for } t \in\left[t_{M}^{o}, t_{M}^{e}\right) .
\end{array}\right.
$$

In the following proof, we discuss the two potential cases of early arrival (i.e., $t \in\left(t_{M}^{b}, t_{M}^{o}\right]$ ) and late arrival (i.e., $t \in\left[t_{M}^{o}, t_{M}^{e}\right)$ ).

\section{(1) Case 1: $t \in\left(t_{M}^{b}, t_{M}^{o}\right]$}

The first commuter departing from home at $t_{M}^{b}$ faces no queue due to his/her early departure from home, but incurs a schedule-delay cost of arriving early, and his/her total net utility in terms of Equation (13) is

$$
U\left(t_{M}^{b}\right)=\int_{0}^{t_{M}^{b}} u_{h}(t) d t+\int_{t_{M}^{b}}^{t_{w}^{d}} u_{w}\left(t-\xi t_{M}^{b}\right) d t-\beta\left(t_{w}^{a^{*}}-t_{M}^{b}\right)+\Lambda_{E}\left(t_{w}^{d}\right),
$$

where $\Lambda_{E}\left(t_{w}^{d}\right)$ is a function with regard to $t_{w}^{d}$, which is given by

$$
\Lambda_{E}\left(t_{w}^{d}\right)=\int_{t_{w}^{d}+T_{E}\left(t_{w}^{d}\right)}^{24} \widehat{u}_{h}(t) d t-\alpha T_{E}\left(t_{w}^{d}\right)-\mu \max \left(0, t_{w}^{d^{*}}-t_{w}^{d}\right)-\lambda \max \left(0, t_{w}^{d}-t_{w}^{d^{*}}\right) .
$$

The total net utility of a commuter departing from home at any time $\tilde{t} \in\left(t_{M}^{b}, t_{M}^{o}\right)$ in the morning is

$U(\tilde{t})=\int_{0}^{\tilde{t}} u_{h}(t) d t+\int_{\tilde{t}+T_{M}(\tilde{t})}^{t_{w}^{d}} u_{w}\left(t-\xi\left(\tilde{t}+T_{M}(\tilde{t})\right)\right) d t-\alpha T_{M}(\tilde{t})-\beta\left(t_{w}^{a^{*}}-\tilde{t}-T_{M}(\tilde{t})\right)+\Lambda_{E}\left(t_{w}^{d}\right)$,

where $\Lambda_{E}\left(t_{w}^{d}\right)$ is given by Equation (A.3).

Consequently, the difference in the total net utilities of the commuters, who depart at $\tilde{t}$ and at $t_{M}^{b}$ is 


$$
\begin{aligned}
U(\tilde{t})-U\left(t_{M}^{b}\right) & =\int_{t_{M}^{b}}^{\tilde{t}} u_{h}(t) d t+\int_{\tilde{t}+T_{M}(\tilde{t})}^{t_{w}^{d}} u_{w}\left(t-\xi\left(\tilde{t}+T_{M}(\tilde{t})\right)\right) d t-\int_{t_{M}^{b}}^{t_{w}^{d}} u_{w}\left(t-\xi t_{M}^{b}\right) d t \\
& -\alpha T_{M}(\tilde{t})-\beta\left(t_{M}^{b}-\tilde{t}-T_{M}(\tilde{t})\right) .
\end{aligned}
$$

Note that the second and third terms on the right-hand side of Equation (A.5) can be respectively expressed as

$$
\begin{aligned}
& \int_{\tilde{t}+T_{M}(\tilde{t})}^{t_{w}^{d}} u_{w}\left(t-\xi\left(\tilde{t}+T_{M}(\tilde{t})\right)\right) d t=\int_{\left(\tilde{t}+T_{M}(\tilde{t})\right)(1-\xi)}^{t_{w}^{d}-\xi\left(\tilde{t}+T_{M}(\tilde{t})\right)} u_{w}(t) d t, \text { and } \\
& \int_{t_{M}^{b}}^{t_{w}^{d}} u_{w}\left(t-\xi t_{M}^{b}\right) d t=\int_{t_{M}^{b}-\xi t_{M}^{t}}^{t_{w}^{d}-\xi t_{M}^{b}} u_{w}(t) d t .
\end{aligned}
$$

According to Equation (A.1) for $t \in\left(t_{M}^{b}, t_{M}^{o}\right]$, the term $\int_{t_{M}^{b}}^{\tilde{t}} u_{h}(t) d t$ in Equation (A.5) can be expressed as

$$
\begin{aligned}
\int_{t_{M}^{b}}^{\tilde{t}} u_{h}(t) d t & \leq \xi \int_{t_{M}^{b}}^{\tilde{t}} u_{w}\left(t_{w}^{d}-\xi t\right) d t-(\xi-1) \int_{t_{M}^{b}}^{\tilde{t}} u_{w}(t-\xi t) d t-\beta\left(\tilde{t}-t_{M}^{b}\right) \\
& =-\int_{t_{w}^{d}-\xi t_{M}^{b}}^{t_{w}^{d}-\xi \tilde{t}} u_{w}(t) d t+\int_{t_{M}^{b}-\xi t_{M}^{b}}^{\tilde{t}-\xi \tilde{t}} u_{w}(t) d t-\beta\left(\tilde{t}-t_{M}^{b}\right) .
\end{aligned}
$$

Substituting Equations (A.6)-(A.8) into (A.5) and carrying out further algebraic operations, we obtain

$$
U(\tilde{t})-U\left(t_{M}^{b}\right) \leq \int_{\left(\tilde{t}+T_{M}(\tilde{t})\right)(1-\xi)}^{t_{w}^{d}-\xi\left(\tilde{t}+T_{M}(\tilde{t})\right)} u_{w}(t) d t-\int_{\tilde{t}-\xi \tilde{t}}^{t_{w}^{d}-\xi \tilde{t}} u_{w}(t) d t+T_{M}(\tilde{t})(\beta-\alpha) .
$$

Note that $\left[\left(\tilde{t}+T_{M}(\tilde{t})\right)(1-\xi), t_{w}^{d}-\xi\left(\tilde{t}+T_{M}(\tilde{t})\right)\right] \subseteq\left[\tilde{t}-\xi \tilde{t}, t_{w}^{d}-\xi \tilde{t}\right]$, so that we have

$$
\int_{\left(\tilde{t}+T_{M}(\tilde{t})\right)(1-\xi)}^{t_{w}^{d}-\xi\left(\tilde{t}+T_{M}(\tilde{t})\right)} u_{w}(t) d t-\int_{\tilde{t}-\xi \tilde{t}}^{t_{w}^{d}-\xi \tilde{t}} u_{w}(t) d t \leq 0 .
$$

As $\alpha>\beta$ and $u_{w}(t) \geq 0$ (i.e., the work activity has a non-negative utility), we have

$$
\int_{\left(\tilde{t}+T_{M}(\tilde{t})(1-\xi)\right.}^{t_{w}^{d}-\xi\left(\tilde{t}+T_{M}(\tilde{t})\right)} u_{w}(t) d t-\int_{\tilde{t}-\xi \tilde{t}}^{t_{w}^{d}-\xi \tilde{t}} u_{w}(t) d t+T_{M}(\tilde{t})(\beta-\alpha) \leq 0 .
$$

However, by the definition of the equilibrium (i.e., Definition 1), we have

$$
U(\tilde{t})-U\left(t_{M}^{b}\right)=0 .
$$

From Equations (A.9) and (A.12), one obtains

$$
\int_{\left(\tilde{t}+T_{M}(\tilde{t})(1-\xi)\right.}^{t_{w}^{d}-\xi\left(\tilde{t}+T_{M}(\tilde{t})\right)} u_{w}(t) d t-\int_{\tilde{t}-\xi \tilde{t}}^{t_{w}^{d}-\xi \tilde{t}} u_{w}(t) d t+T_{M}(\tilde{t})(\beta-\alpha) \geq 0 .
$$

According to Equations (A.11) and (A.13), the following equation must be satisfied: 


$$
T_{M}(\tilde{t})=0, \forall \tilde{t} \in\left(t_{M}^{b}, t_{M}^{o}\right]
$$

This condition contradicts the assumption that a queue exists at the bottleneck.

\section{(2) Case 2: $t \in\left[t_{M}^{o}, t_{M}^{e}\right)$}

The last commuter who departs at $t_{M}^{e}$ faces no queue due to his/her late departure from home, but suffers a schedule delay by arriving late. According to Equation (13), the total net utility received by the last commuter is

$$
U\left(t_{M}^{e}\right)=\int_{0}^{t_{M}^{e}} u_{h}(t) d t+\int_{t_{M}^{e}}^{t_{w}^{d}} u_{w}\left(t-\xi t_{M}^{e}\right) d t-\gamma\left(t_{M}^{e}-t_{w}^{a^{*}}\right)+\Lambda_{E}\left(t_{w}^{d}\right)
$$

where $\Lambda_{E}\left(t_{w}^{d}\right)$ is given by Equation (A.3).

The total net utility of the commuters departing from home at any time $\tilde{t} \in\left[t_{M}^{o}, t_{M}^{e}\right)$ is

$$
U(\tilde{t})=\int_{0}^{\tilde{t}} u_{h}(t) d t+\int_{\tilde{t}+T_{M}(\tilde{t})}^{t_{w}^{d}} u_{w}\left(t-\xi\left(\tilde{t}+T_{M}(\tilde{t})\right)\right) d t-\alpha T_{M}(\tilde{t})-\gamma\left(\tilde{t}+T_{M}(\tilde{t})-t_{w}^{a^{*}}\right)+\Lambda_{E}\left(t_{w}^{d}\right) .
$$

Hence, we obtain the difference of the total net utilities as follows:

$$
\begin{gathered}
U\left(t_{M}^{e}\right)-U(\tilde{t})=\int_{\tilde{t}}^{t_{M}^{e}} u_{h}(t) d t+\int_{t_{M}^{e}}^{t_{w}^{d}} u_{w}\left(t-\xi t_{M}^{e}\right) d t-\int_{\tilde{t}+T_{M}(\tilde{t})}^{t_{w}^{d}} u_{w}\left(t-\xi\left(\tilde{t}+T_{M}(\tilde{t})\right)\right) d t+\alpha T_{M}(\tilde{t})-\gamma\left(t_{M}^{e}-\tilde{t}-T_{M}(\tilde{t})\right) \\
=\int_{\tilde{t}}^{t_{M}^{e}} u_{h}(t) d t+\int_{t_{M}^{e}-\xi t_{M}^{e}}^{t_{w}^{d}-\xi t_{M}^{e}} u_{w}(t) d t-\int_{\left(\tilde{t}+T_{M}(\tilde{t})\right)(1-\xi)}^{t_{w}^{d}-\xi\left(\tilde{t}+T_{M}(\tilde{t})\right)} u_{w}(t) d t+\alpha T_{M}(\tilde{t})-\gamma\left(t_{M}^{e}-\tilde{t}-T_{M}(\tilde{t})\right) .
\end{gathered}
$$

According to Equation (A.1) for $t \in\left[t_{M}^{o}, t_{M}^{e}\right.$ ), the term $\int_{\tilde{t}}^{t_{M}^{e}} u_{h}(t) d t$ in Equation (A.17) can be expressed as

$$
\begin{aligned}
\int_{\tilde{t}}^{t_{M}^{e}} u_{h}(t) d t & \geq \xi \int_{\tilde{t}}^{t_{M}^{e}} u_{w}\left(t_{w}^{d}-\xi t\right) d t-(\xi-1) \int_{\tilde{t}}^{t_{M}^{e}} u_{w}(t-\xi t) d t+\gamma\left(t_{M}^{e}-\tilde{t}\right) \\
& =-\int_{t_{w}^{d}-\xi \tilde{t}}^{t_{w}^{d}-\xi t_{M}^{e}} u_{w}(t) d t+\int_{\tilde{t}-\xi \tilde{t}}^{t_{M}^{e}-\xi t_{M}^{e}} u_{w}(t) d t+\gamma\left(t_{M}^{e}-\tilde{t}\right) .
\end{aligned}
$$

From Equations (A.17) and (A.18) we obtain

$$
U\left(t_{M}^{e}\right)-U(\tilde{t}) \geq \int_{\tilde{t}-\xi \tilde{t}}^{t_{w}^{d}-\xi \tilde{t}} u_{w}(t) d t-\int_{\left(\tilde{t}+T_{M}(\tilde{t})\right)(1-\xi)}^{t_{w}^{d}-\xi\left(\tilde{t}+T_{M}(\tilde{t})\right)} u_{w}(t) d t+(\alpha+\gamma) T_{M}(\tilde{t})
$$

According to Equations (A.10) and (A.19) we have

$$
\int_{\tilde{t}-\xi \tilde{t}}^{t_{w}^{d}-\xi \tilde{t}} u_{w}(t) d t-\int_{\left(\tilde{t}+T_{M}(\tilde{t})\right)(1-\xi)}^{t_{w}^{d}-\xi\left(\tilde{t}+T_{M}(\tilde{t})\right)} u_{w}(t) d t+(\alpha+\gamma) T_{M}(\tilde{t}) \geq 0
$$

However, by the equilibrium condition $U\left(t_{M}^{e}\right)-U(\tilde{t})=0$ we have 


$$
\int_{\tilde{t}-\xi \tilde{t}}^{t_{w}^{d}-\xi \tilde{t}} u_{w}(t) d t-\int_{\left(\tilde{t}+T_{M}(\tilde{t})\right)(1-\xi)}^{t_{w}^{d}-\xi\left(\tilde{t}+T_{M}(\tilde{t})\right)} u_{w}(t) d t+(\alpha+\gamma) T_{M}(\tilde{t}) \leq 0
$$

Consequently, we obtain

$$
T_{M}(\tilde{t})=0, \text { for } \tilde{t} \in\left[t_{M}^{o}, t_{M}^{e}\right) .
$$

This condition contradicts the assumption that a queue exists at the bottleneck.

(Sufficiency) We need to prove that Equation (18) implies that there is a queue at the bottleneck. Again, the contradiction method is used. Suppose that no queue appears at the bottleneck for any $t \in\left(t_{M}^{b}, t_{M}^{e}\right)$, i.e., $T_{M}(t)=0, \forall t \in\left(t_{M}^{b}, t_{M}^{e}\right)$. Two cases are discussed as follows.

(1) Case 1: $t \in\left(t_{M}^{b}, t_{M}^{o}\right]$

Referring to Equation (A.5), if there is no bottleneck congestion (i.e., $T_{M}(\tilde{t})=0$ ), then the difference in the total net utilities of the commuters departing at $\tilde{t}$ and $t_{M}^{b}$ is

$$
\begin{aligned}
& U(\tilde{t})-U\left(t_{M}^{b}\right)=\int_{t_{M}^{b}}^{\tilde{t}} u_{h}(t) d t+\int_{\tilde{t}}^{t_{w}^{d}} u_{w}(t-\xi \tilde{t}) d t-\int_{t_{M}^{b}}^{t_{w}^{d}} u_{w}\left(t-\xi t_{M}^{b}\right) d t-\beta\left(t_{M}^{b}-\tilde{t}\right) \\
& =\int_{t_{M}^{b}}^{\tilde{t}} u_{h}(t) d t+\int_{\tilde{t}-\xi \tilde{t}}^{t_{w}^{d}-\xi \tilde{t}} u_{w}(t) d t-\int_{t_{M}^{b}-\xi t_{M}^{b}}^{t_{w}^{d}-\xi t_{M}^{b}} u_{w}(t) d t-\beta\left(t_{M}^{b}-\tilde{t}\right) \\
& =\int_{t_{M}^{b}}^{\tilde{t}} u_{h}(t) d t-\int_{t_{M}^{b}-\xi t_{M}^{t^{u}}}^{\tilde{t}-\xi \tilde{t}} u_{w}(t) d t-\int_{t_{w}^{d}-\xi \tilde{t}}^{t_{w}^{d}-\xi t_{M}^{b}} u_{w}(t) d t-\beta\left(t_{M}^{b}-\tilde{t}\right) .
\end{aligned}
$$

According to Equation (A.8), Equation (A.23) can be further written as

$$
\begin{aligned}
& U(\tilde{t})-U\left(t_{M}^{b}\right)=\int_{t_{M}^{b}}^{\tilde{t}} u_{h}(t) d t-\xi \int_{t_{M}^{b}}^{\tilde{t}} u_{w}\left(t_{w}^{d}-\xi t\right) d t+(\xi-1) \int_{t_{M}^{b}}^{\tilde{t}} u_{w}(t-\xi t) d t-\beta\left(t_{M}^{b}-\tilde{t}\right) \\
& =\int_{t_{M}^{b}}^{\tilde{t}}\left(u_{h}(t)-\xi u_{w}\left(t_{w}^{d}-\xi t\right)+(\xi-1) u_{w}(t-\xi t)+\beta\right) d t .
\end{aligned}
$$

At the equilibrium state, $U(\tilde{t})=U\left(t_{M}^{b}\right)$ holds. Thus, we have

$$
u_{h}(t)-\xi u_{w}\left(t_{w}^{d}-\xi t\right)+(\xi-1) u_{w}(t-\xi t)+\beta=0 .
$$

This condition contradicts the assumption that $u_{h}(t)>\xi u_{w}\left(t_{w}^{d}-\xi t\right)-(\xi-1) u_{w}(t-\xi t)-\beta$.

(2) Case 2: $t \in\left[t_{M}^{o}, t_{M}^{e}\right)$

According to Equation (A.17), when there is no bottleneck congestion (i.e., $T_{M}(\tilde{t})=0$ ), the difference in the total net utilities of the commuters departing at times $\tilde{t}$ and $t_{M}^{e}$ is 


$$
\begin{gathered}
U\left(t_{M}^{e}\right)-U(\tilde{t})=\int_{\tilde{t}}^{t_{M}^{e}} u_{h}(t) d t+\int_{t_{M}^{e}-\xi \xi_{M}^{e}}^{t_{w}^{d}-\xi t_{M}^{e}} u_{w}(t) d t-\int_{\tilde{t}(1-\xi)}^{t_{w}^{d}-\xi \tilde{t}} u_{w}(t) d t-\gamma\left(t_{M}^{e}-\tilde{t}\right) \\
=\int_{\tilde{t}}^{t_{M}^{e}} u_{h}(t) d t+\int_{t_{w}^{d}-\xi \tilde{t}}^{t_{w}^{d}-\xi t_{M}^{e}} u_{w}(t) d t-\int_{\tilde{t}-\xi \tilde{t}}^{t_{M}^{e}-\xi t_{M}^{e}} u_{w}(t) d t-\gamma\left(t_{M}^{e}-\tilde{t}\right) .
\end{gathered}
$$

From Equation (A.18), Equation (A.26) can be rewritten as

$$
\begin{gathered}
U\left(t_{M}^{e}\right)-U(\tilde{t})=\int_{\tilde{t}}^{t_{M}^{e}} u_{h}(t) d t+\xi \int_{\tilde{t}}^{t_{M}^{e}} u_{w}\left(t_{w}^{d}-\xi t\right) d t-(\xi-1) \int_{\tilde{t}}^{t_{M}^{e}} u_{w}(t-\xi t) d t+\gamma\left(t_{M}^{e}-\tilde{t}\right) \\
=\int_{\tilde{t}}^{t_{M}^{e}}\left(u_{h}(t)+\xi u_{w}\left(t_{w}^{d}-\xi t\right)-(\xi-1) u_{w}(t-\xi t)+\gamma\right) d t .
\end{gathered}
$$

By the equilibrium condition $U(\tilde{t})=U\left(t_{M}^{e}\right)$, we obtain

$$
u_{h}(t)+\xi u_{w}\left(t_{w}^{d}-\xi t\right)-(\xi-1) u_{w}(t-\xi t)+\gamma=0 .
$$

This condition contradicts the assumption that $u_{h}(t)<\xi u_{w}\left(t_{w}^{d}-\xi t\right)-(\xi-1) u_{w}(t-\xi t)+\gamma$.

This completes the proof of this proposition. 


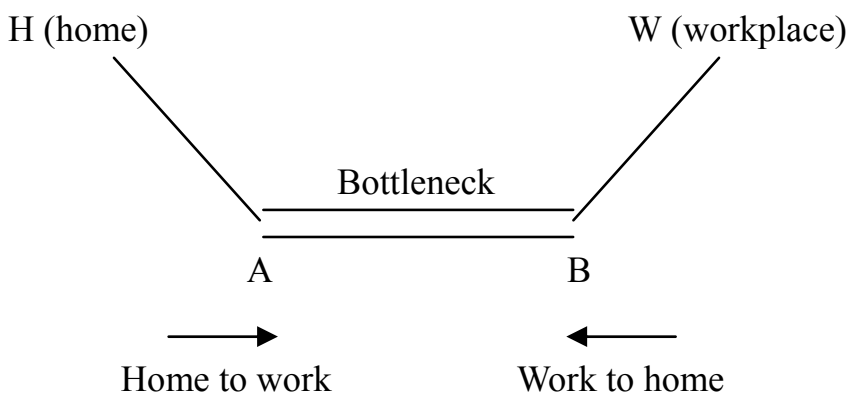

Figure 1. The setting of the model. 


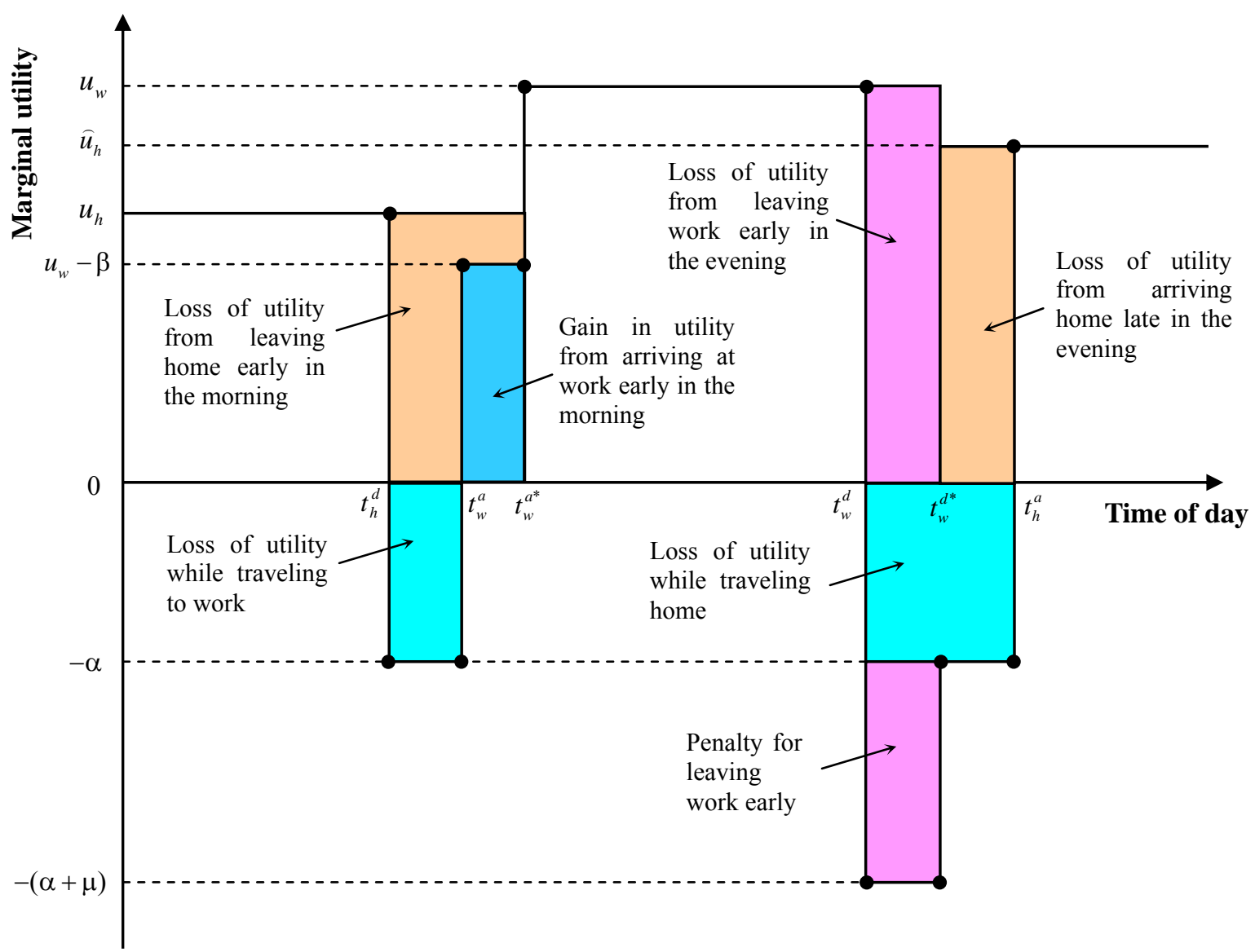

Figure 2. An example of the net utility function with constant marginal activity utilities.

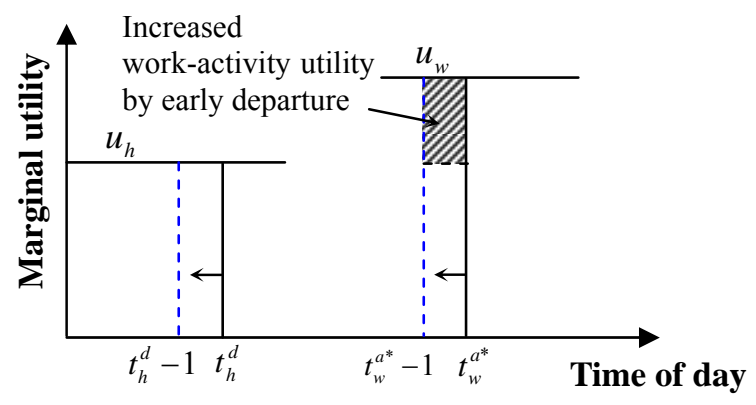

(a)

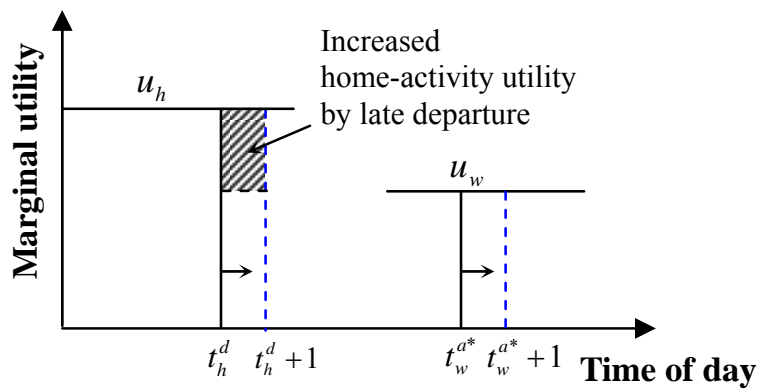

(b)

Figure 3. Utilities of home and work activities against morning departure-time choices: (a)

$$
u_{h} \leq u_{w}-\beta ; \text { (b) } u_{h} \geq u_{w}+\gamma \text {. }
$$




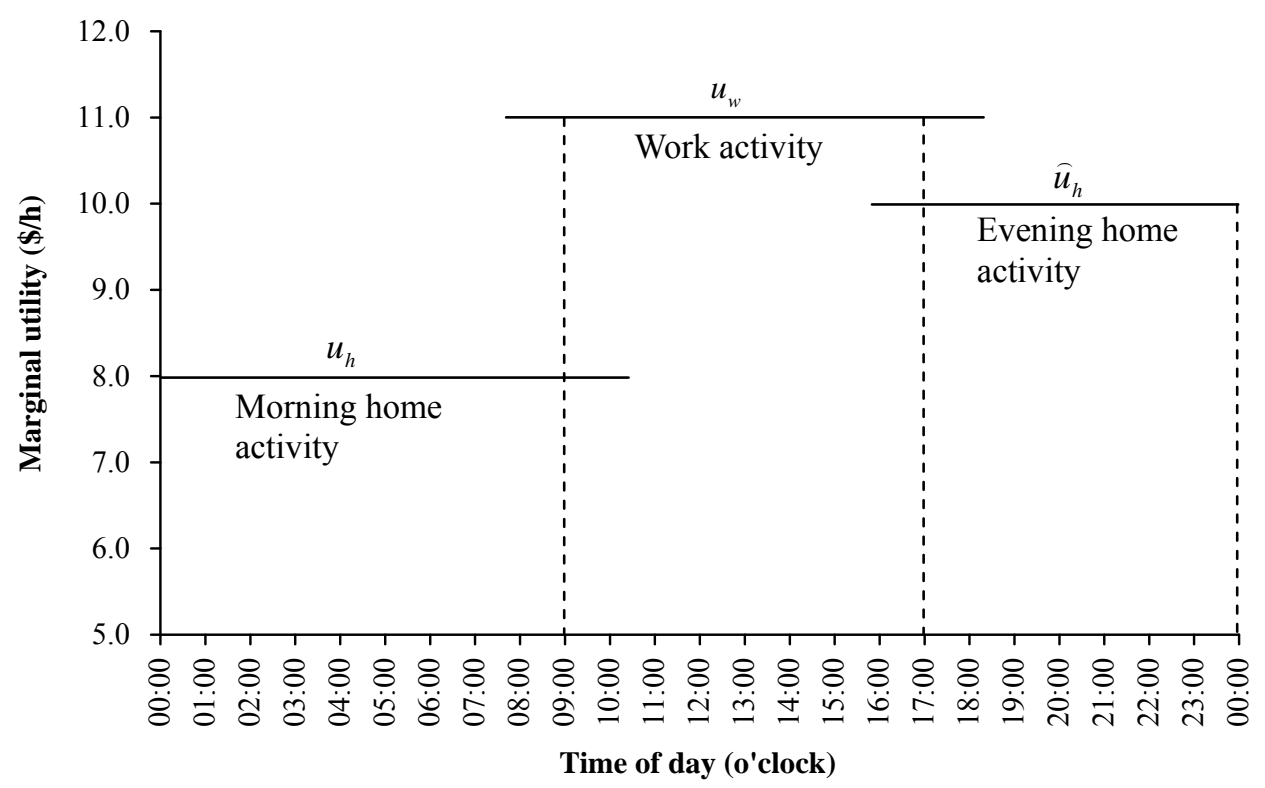

Figure 4. Constant marginal utilities for home and work activities. 


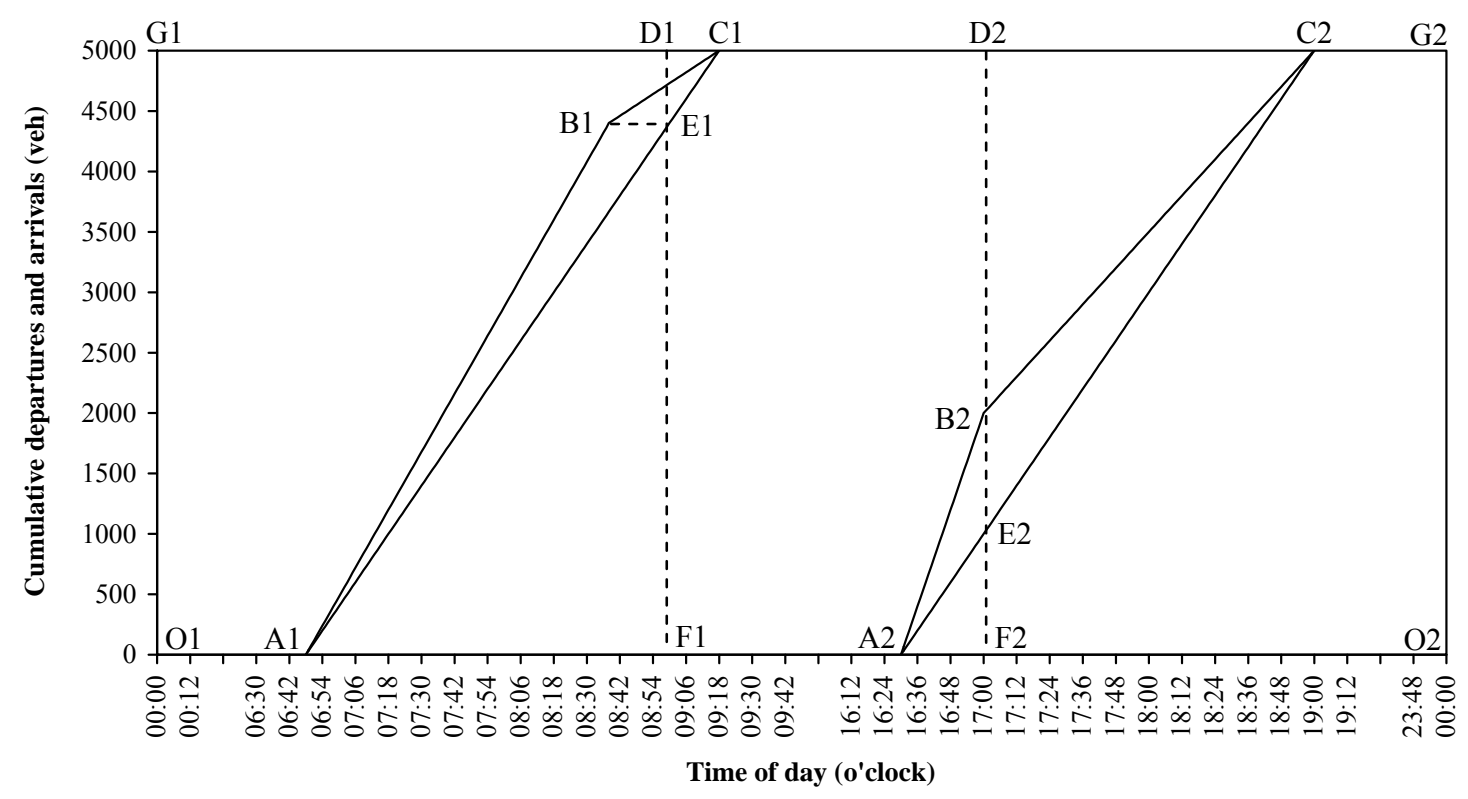

(a)

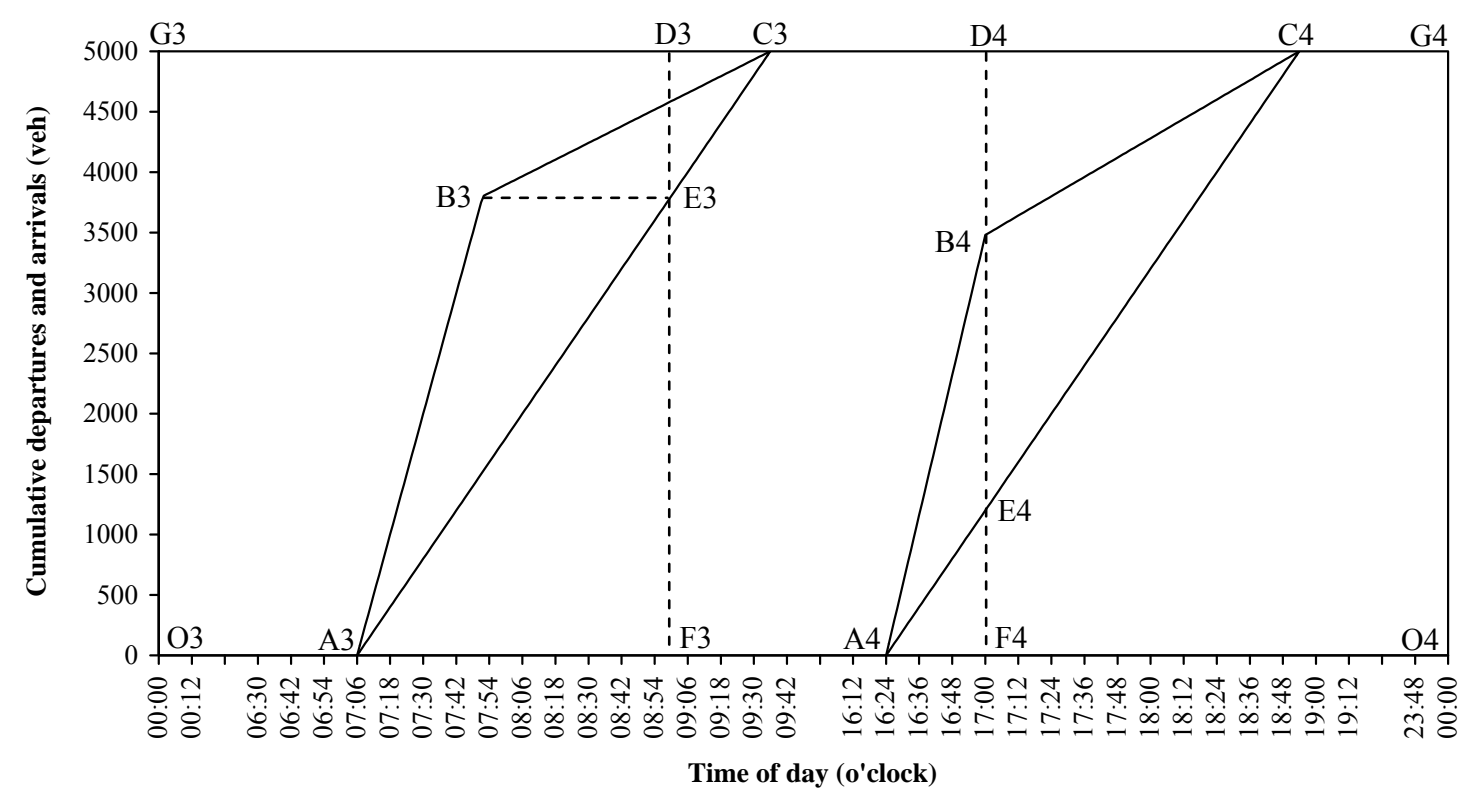

(b)

Figure 5. Cumulative departures and arrivals over times of a day: (a) activity-based model; (b) trip-based model. 


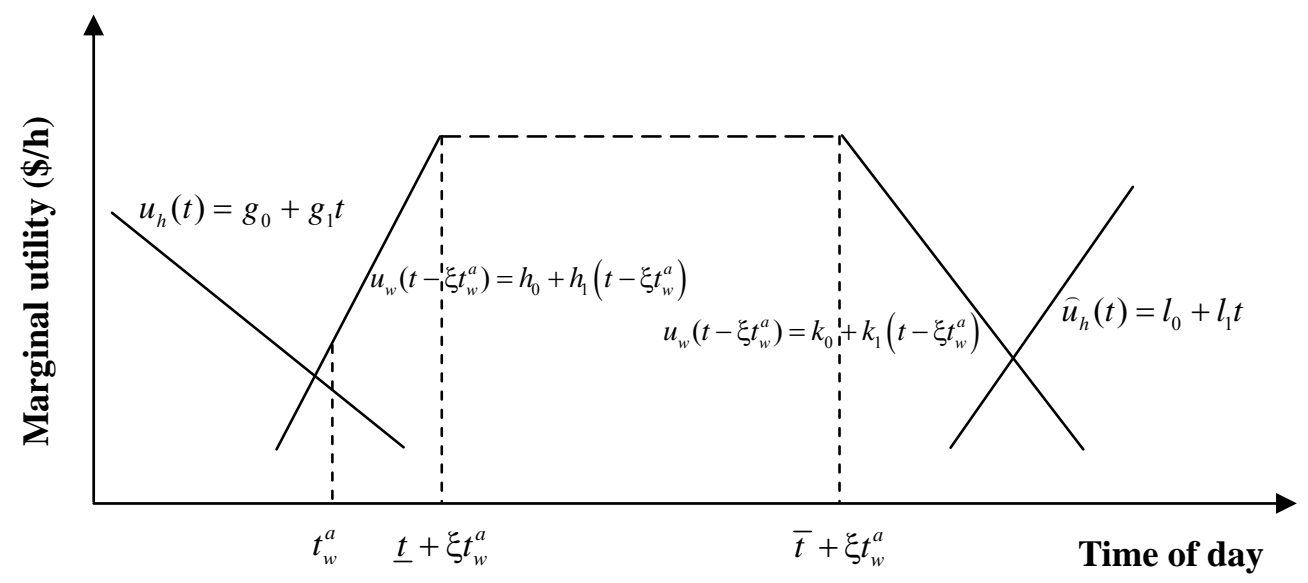

Figure 6. Linear marginal utility functions for home and work activities (see Jenelius et al., 2011 and Jenelius, 2012).

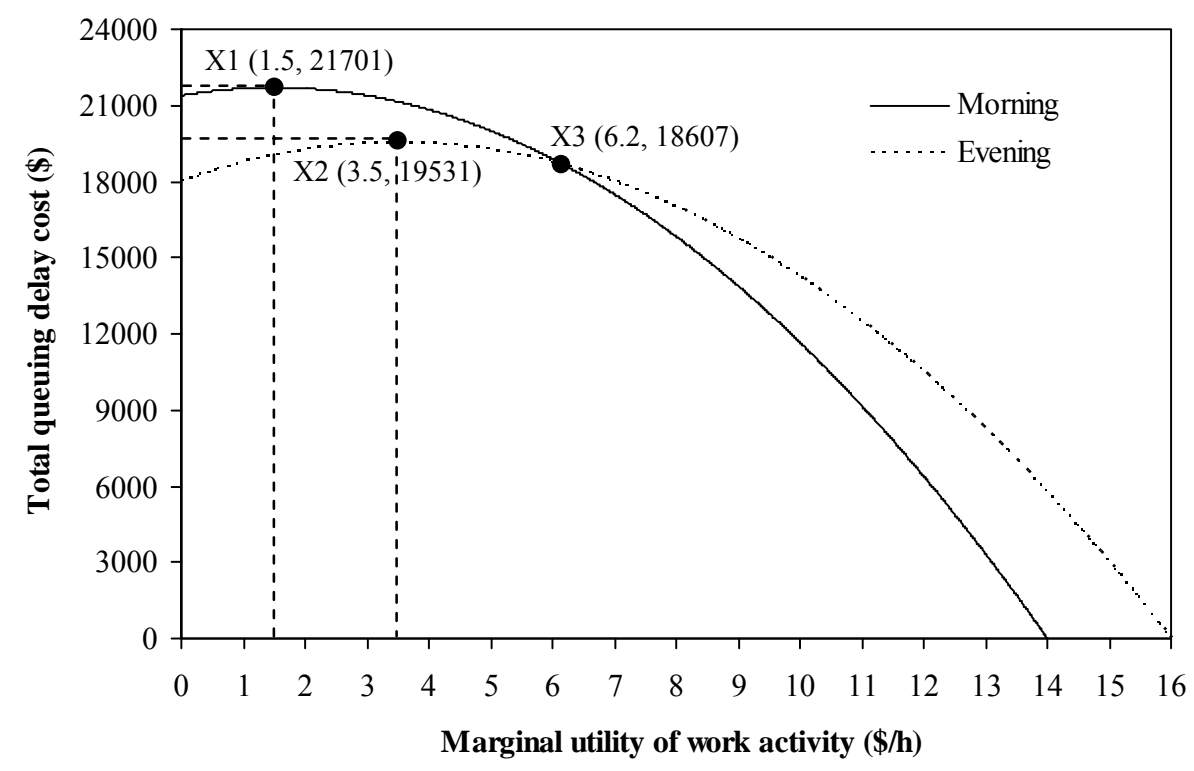

Figure 7. Effects of marginal utility of work activity on total queuing-delay costs at the bottleneck in the morning and evening peaks. 


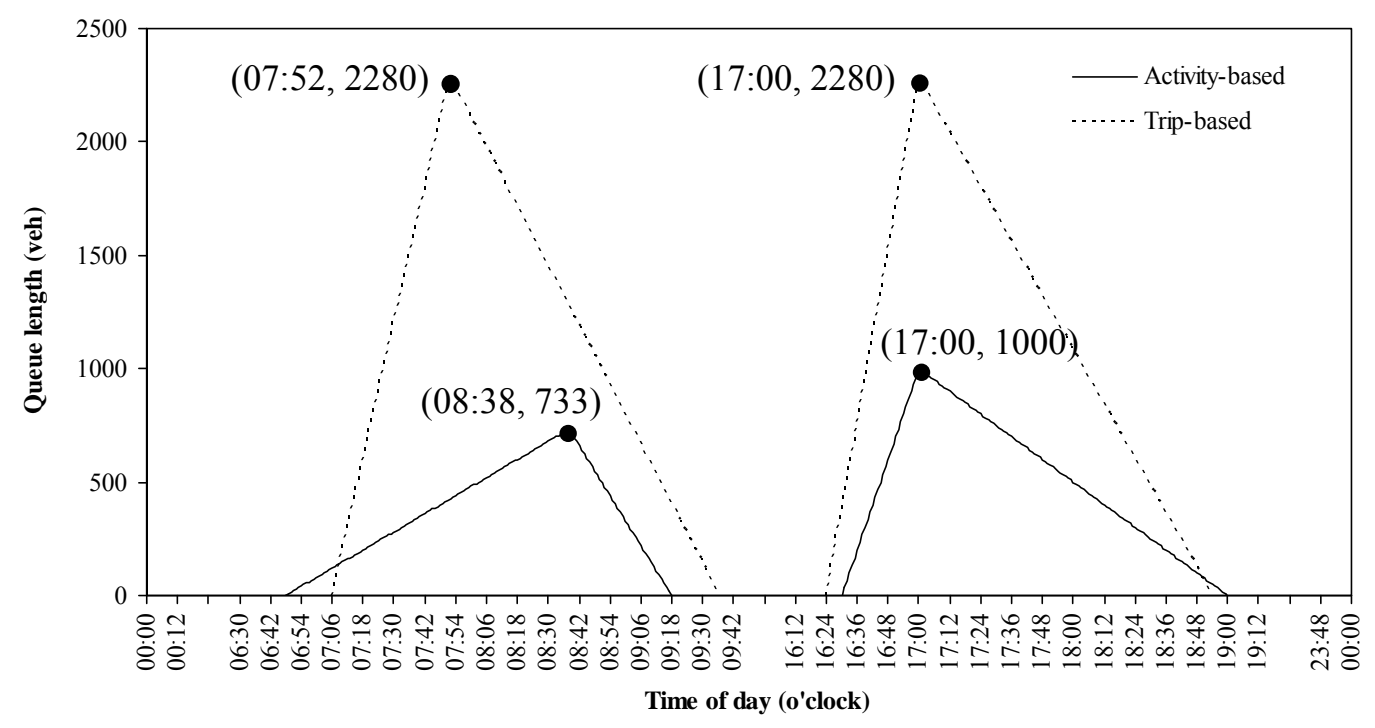

Figure 8. Queue lengths over times of a day under activity- and trip-based approaches.

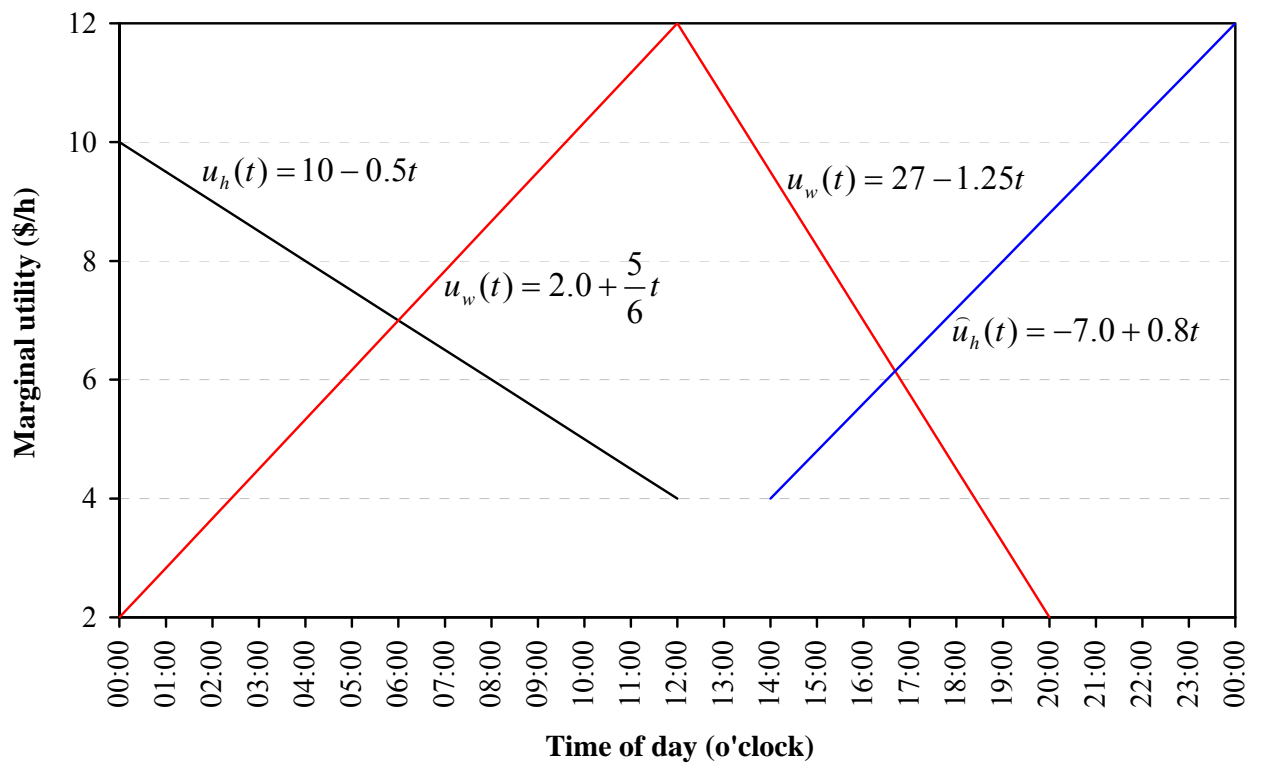

Figure 9. Linear marginal utilities for home and work activities. 


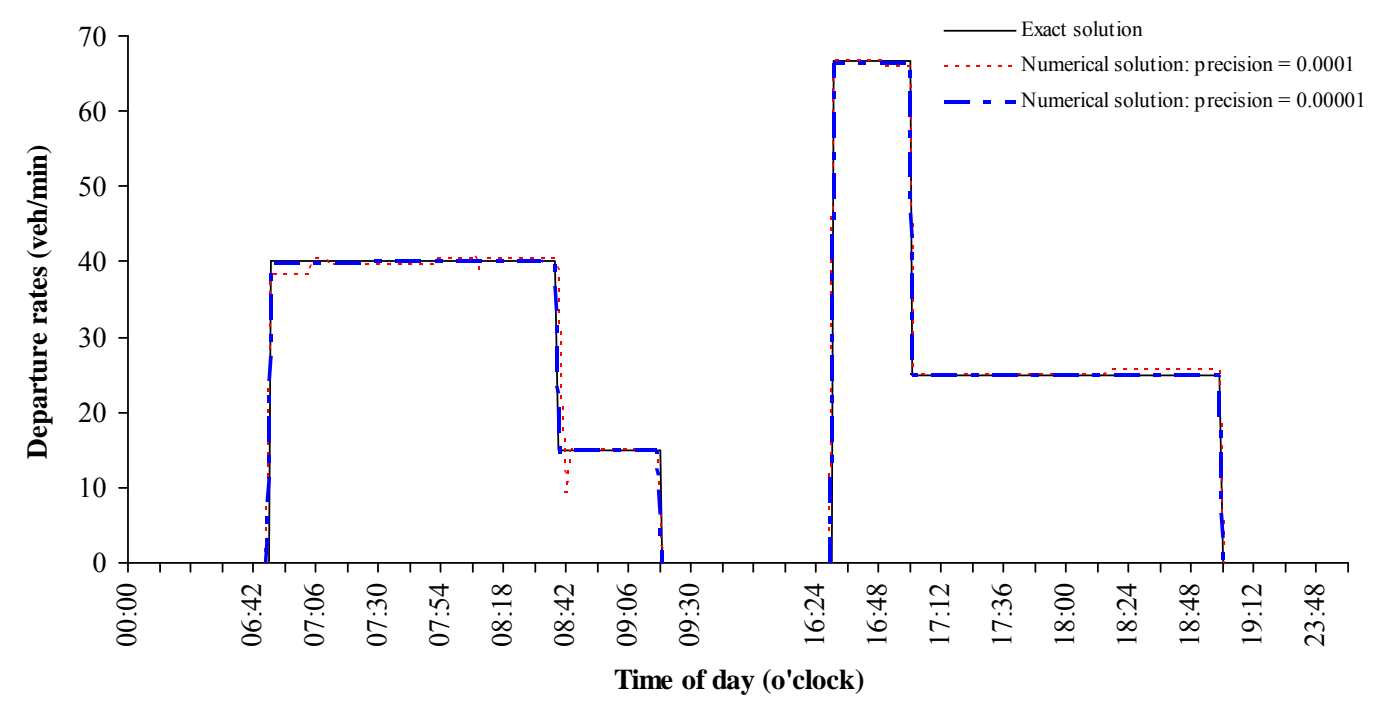

Figure 10. Numerical solutions for Scenario 1 at different levels of precision.

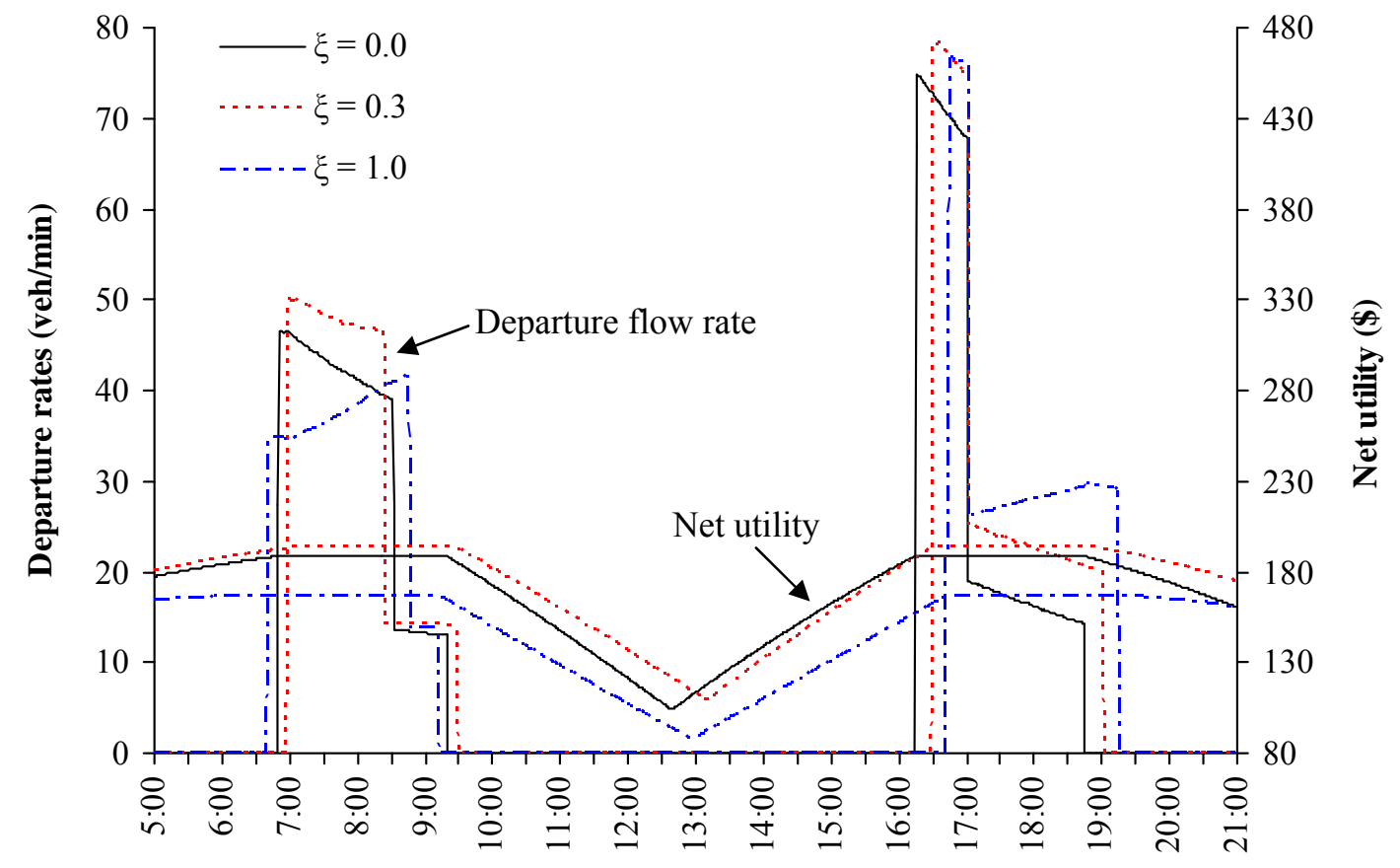

Time of day (o'clock)

Figure 11. Departure flow rates in the morning and evening peaks and net utility for different flexibility parameters. 


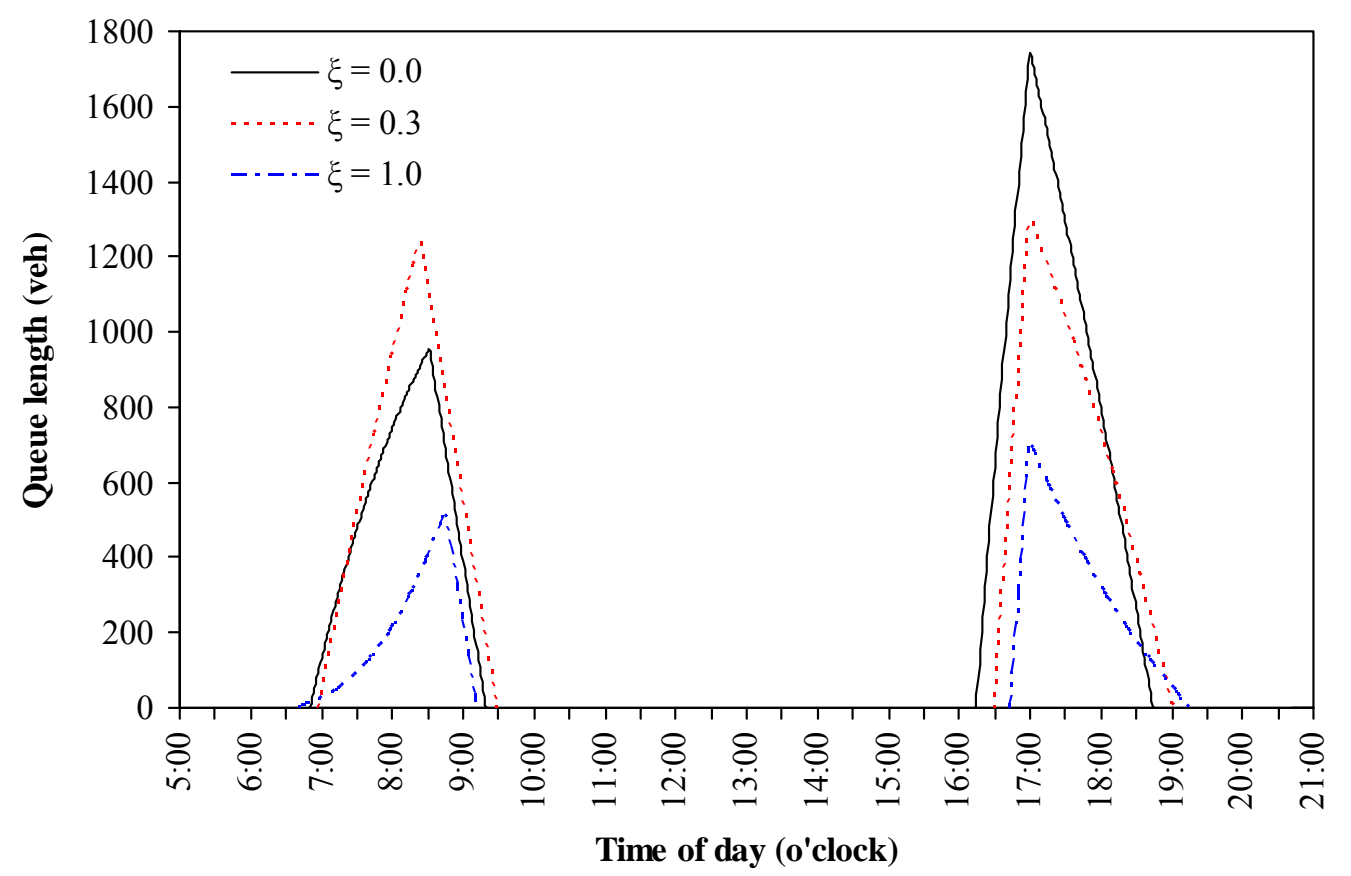

Figure 12. Queue lengths during morning and evening commutes for different flexibility parameters.

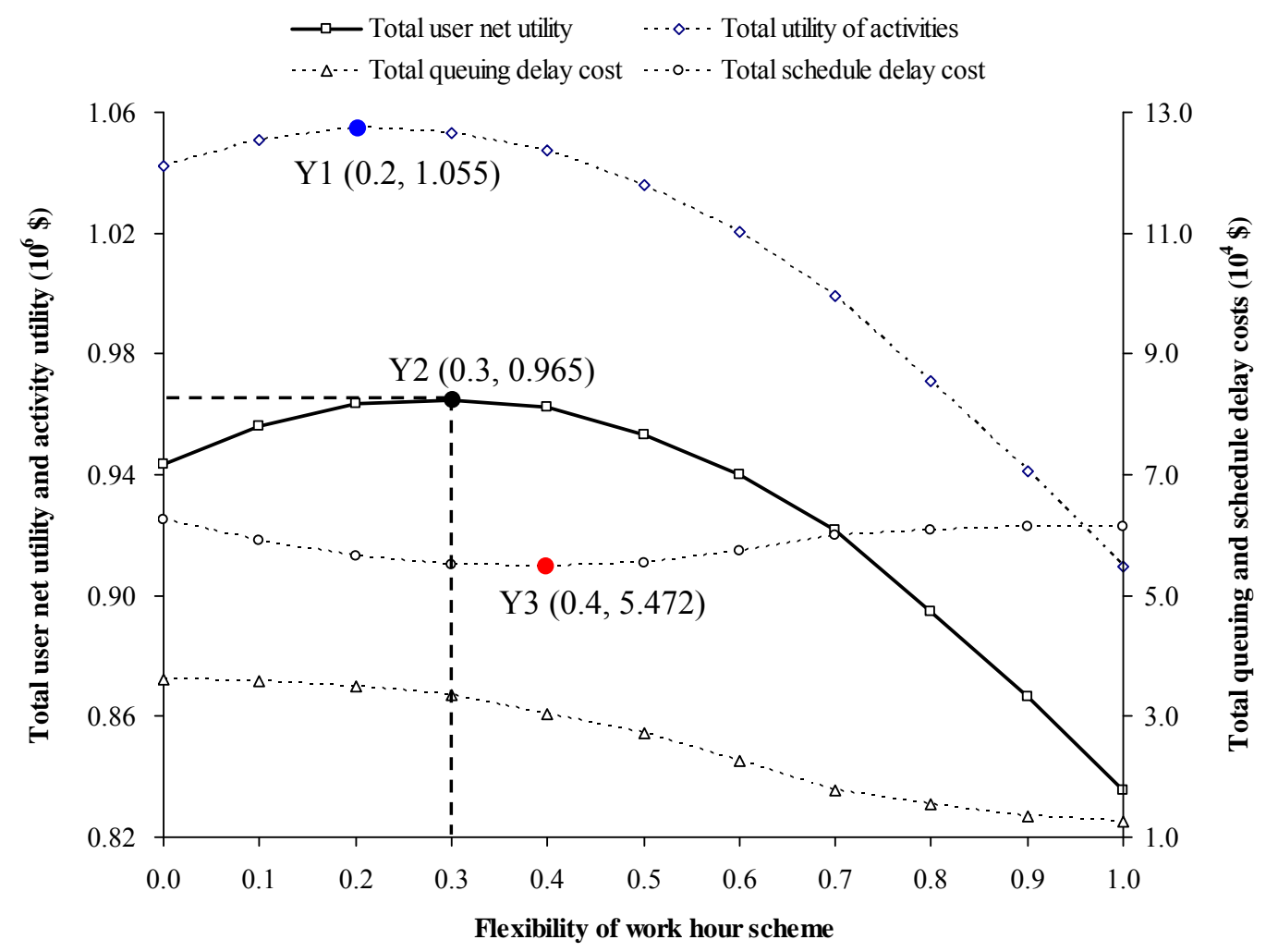

Figure 13. Effects of flexibility parameter on performance of transportation system. 
Table 1 Input data for the model parameters.

\begin{tabular}{clc}
\hline Symbol & \multicolumn{1}{c}{ Definition } & Baseline \\
& & value \\
\hline$N$ & number of commuters & 5,000 \\
$S$ & capacity of bottleneck $(\mathrm{veh} / \mathrm{h})$ & 2,000 \\
$t_{w}^{a^{*}}$ & preferred arrival time at work in the morning & $09: 00$ \\
$t_{w}^{d^{*}}$ & preferred departure time from work in the evening & $17: 00$ \\
$u_{w}$ & constant marginal utility of work activity $(\$ / \mathrm{h})$ & 11.0 \\
$u_{h}$ & constant marginal utility of home activity pre-work $(\$ / \mathrm{h})$ & 8.0 \\
$\widehat{u}_{h}$ & constant marginal utility of home activity post-work $(\$ / \mathrm{h})$ & 10.0 \\
$\alpha$ & unit cost of travel time $(\$ / \mathrm{h})$ & 10.0 \\
$\beta$ & unit cost of arriving early $(\$ / \mathrm{h})$ & 6.0 \\
$\gamma$ & unit cost of arriving late $(\$ / \mathrm{h})$ & 19.0 \\
$\mu$ & unit cost of departing early $(\$ / \mathrm{h})$ & 19.0 \\
$\lambda$ & unit cost of departing late $(\$ / \mathrm{h})$ & 6.0 \\
\hline
\end{tabular}


Table 2 Analytical solutions for time allocation under activity- and trip-based approaches.

$$
\text { Activity-based approach }
$$

Trip-based approach

Average travel

time in the

morning peak

$$
\frac{N}{2 S} \frac{\left(u_{h}-u_{w}+\beta\right)\left(u_{w}-u_{h}+\gamma\right)}{(\beta+\gamma)\left(\alpha+u_{h}\right)}
$$

$$
\frac{N}{2 S} \frac{\beta \gamma}{\alpha(\beta+\gamma)}
$$

$\operatorname{ATT}_{M} \quad(\mathrm{~h})$

Average travel

time in the

evening peak

$$
\frac{N}{2 S} \frac{\left(\widehat{u}_{h}-u_{w}+\lambda\right)\left(u_{w}-\widehat{u}_{h}+\mu\right)}{(\mu+\lambda)\left(\alpha+\widehat{u}_{h}\right)}
$$

$$
\frac{N}{2 S} \frac{\mu \lambda}{\alpha(\mu+\lambda)}
$$

$\operatorname{ATT}_{E}$ (h)

Average duration

for morning home

$$
t_{w}^{a^{*}}+\frac{N}{2 S}\left(\frac{2 u_{h}-2 u_{w}+\beta-\gamma}{\beta+\gamma}\right)
$$

activity $A D_{h, M}$

(h) $\quad-\frac{N}{2 S} \frac{\left(u_{h}-u_{w}+\beta\right)\left(u_{w}-u_{h}+\gamma\right)}{(\beta+\gamma)\left(\alpha+u_{h}\right)}$

$$
t_{w}^{a^{*}}+\frac{N}{2 S} \frac{\alpha \beta-\alpha \gamma-\beta \gamma}{\alpha(\beta+\gamma)}
$$

Average duration

for evening home

activity $A D_{h, E}$

(h)

$$
24-t_{w}^{d^{*}}-\frac{N}{2 S} \frac{2 u_{w}-2 \widehat{u}_{h}+\mu-\lambda}{\mu+\lambda}
$$

$$
24-t_{w}^{d^{*}}-\frac{N}{2 S} \frac{\mu-\lambda}{\mu+\lambda}
$$

Average duration

for work activity

$A D_{w}$ (h)

$$
\begin{aligned}
& t_{w}^{d^{*}}-t_{w}^{a^{*}}+\frac{N}{S} \frac{u_{w}-u_{h}+\gamma}{\beta+\gamma} \\
& -\frac{N}{2 S} \frac{\left(\widehat{u}_{h}-u_{w}+\lambda\right)\left(2 \alpha+\widehat{u}_{h}+u_{w}+\mu\right)}{\left(\alpha+\widehat{u}_{h}\right)(\mu+\lambda)}
\end{aligned} \quad t_{w}^{d^{*}}-t_{w}^{a^{*}}+\frac{N}{S} \frac{\gamma}{\beta+\gamma}-\frac{N}{2 S} \frac{\lambda(2 \alpha+\mu)}{\alpha(\mu+\lambda)}
$$


Table 3 Analytical solutions for system performances under activity-based and trip-based approaches.

\section{Activity-based approach}

Trip-based approach

\begin{tabular}{|c|c|c|}
\hline Total travel-time & & \\
\hline cost in the & $\alpha N^{2}\left(u_{h}-u_{w}+\beta\right)\left(u_{w}-u_{h}+\gamma\right)$ & $N^{2} \beta \gamma$ \\
\hline morning peak & $(\beta+\gamma)\left(\alpha+u_{h}\right)$ & $\overline{2 S} \overline{\beta+\gamma}$ \\
\hline
\end{tabular}

$\operatorname{TTC}_{M}(\$)$

Total travel-time

cost in the

evening peak

$$
\frac{\alpha N^{2}}{2 S} \frac{\left(\widehat{u}_{h}-u_{w}+\lambda\right)\left(u_{w}-\widehat{u}_{h}+\mu\right)}{(\mu+\lambda)\left(\alpha+\widehat{u}_{h}\right)}
$$

$\frac{N^{2}}{2 S} \frac{\mu \lambda}{\mu+\lambda}$

$\operatorname{TTC}_{E}(\$)$

Total

schedule-delay

cost in the

morning peak

$$
\frac{N^{2}}{2 S(\beta+\gamma)^{2}}\left(\beta\left(u_{w}-u_{h}+\gamma\right)^{2}+\gamma\left(u_{h}-u_{w}+\beta\right)^{2}\right) \quad \frac{N^{2}}{2 S} \frac{\beta \gamma}{\beta+\gamma}
$$

$S D C_{M} \quad(\$)$

Total

schedule-delay

cost in the

evening peak

$$
\frac{N^{2}}{2 S\left(\alpha+\widehat{u}_{h}\right)(\mu+\lambda)^{2}}\left(\begin{array}{l}
\mu\left(\alpha+\mu+u_{w}\right)\left(\widehat{u}_{h}-u_{w}+\lambda\right)^{2} \\
+\lambda\left(\alpha-\lambda+u_{w}\right)\left(u_{w}-\widehat{u}_{h}+\mu\right)^{2}
\end{array}\right) \quad \frac{N^{2}}{2 S} \frac{\mu \lambda}{\mu+\lambda}
$$

$S D C_{E}(\$)$

Total utility of morning home activity $T U_{h, M}$

$$
N u_{h}\left(\begin{array}{l}
t_{w}^{a^{*}}+\frac{N}{2 S} \frac{2 u_{h}-2 u_{w}+\beta-\gamma}{\beta+\gamma} \\
-\frac{N}{2 S} \frac{\left(u_{h}-u_{w}+\beta\right)\left(u_{w}-u_{h}+\gamma\right)}{(\beta+\gamma)\left(\alpha+u_{h}\right)}
\end{array}\right)
$$$$
N u_{h}\left(t_{w}^{a^{*}}+\frac{N}{2 S} \frac{\alpha \beta-\alpha \gamma-\beta \gamma}{\alpha(\beta+\gamma)}\right)
$$

Total utility of evening home activity $T U_{h, E}$

$$
N \hat{u}_{h}\left(24-t_{w}^{d^{*}}-\frac{N}{2 S} \frac{2 u_{w}-2 \widehat{u}_{h}+\mu-\lambda}{\mu+\lambda}\right) \quad N \widehat{u}_{h}\left(24-t_{w}^{d^{*}}-\frac{N}{2 S} \frac{\mu-\lambda}{\mu+\lambda}\right)
$$

Total utility of work activity $T U_{w}$

$$
N u_{w}\left(\begin{array}{l}
t_{w}^{d^{*}}-t_{w}^{a^{*}}+\frac{N}{S} \frac{u_{w}-u_{h}+\gamma}{\beta+\gamma} \\
-\frac{N}{2 S} \frac{\left(\widehat{u}_{h}-u_{w}+\lambda\right)\left(2 \alpha+\widehat{u}_{h}+u_{w}+\mu\right)}{\left(\alpha+\widehat{u}_{h}\right)(\mu+\lambda)}
\end{array}\right)
$$$$
N u_{w}\left(\begin{array}{l}
t_{w}^{d^{*}}-t_{w}^{a^{*}}+\frac{N}{S} \frac{\gamma}{\beta+\gamma} \\
-\frac{N}{2 S} \frac{\lambda(2 \alpha+\mu)}{\alpha(\mu+\lambda)}
\end{array}\right)
$$

Total net utility of the system

$$
T U_{h, M}+T U_{h, E}+T U_{w}-T T C_{M}-T T C_{E}-S D C_{M}-S D C_{E}
$$

during a day (\$) 
Table 4 The relationship between $t_{w}^{a}$ and $t_{w}^{d}$.

\begin{tabular}{lccc}
\hline \multirow{2}{*}{$t_{w}^{a}$ belongs to } & \multicolumn{3}{c}{$t_{w}^{d}$ belongs to } \\
\cline { 2 - 4 } & {$\left[0, \underline{t}+\xi t_{w}^{a}\right]$} & {$\left[\underline{t}+\xi t_{w}^{a}, \bar{t}+\xi t_{w}^{a}\right]$} & {$\left[\bar{t}+\xi t_{w}^{a}, 24\right]$} \\
\hline$\left[0, \underline{t}+\xi t_{w}^{a}\right]$ & $\sqrt{ }$ & $\sqrt{ }$ & $\sqrt{ }$ \\
{$\left[\underline{t}+\xi t_{w}^{a}, \bar{t}+\xi t_{w}^{a}\right]$} & & & $\sqrt{ }$ \\
{$\left[\bar{t}+\xi t_{w}^{a}, 24\right]$} & & $\sqrt{ }$ \\
\hline
\end{tabular}

Table 5 Time allocation under activity- and trip-based approaches.

\begin{tabular}{lcc}
\hline & $\begin{array}{c}\text { Activity-based } \\
\text { approach }\end{array}$ & $\begin{array}{c}\text { Trip-based } \\
\text { approach }\end{array}$ \\
\hline Average travel time in the morning peak (h) & 0.18 & 0.57 \\
Average travel time in the evening peak (h) & 0.25 & 0.57 \\
Average duration for morning home activity (h) & 7.87 & 7.78 \\
Average duration for evening home activity (h) & 6.25 & 6.35 \\
Average duration for work activity (h) & 9.45 & 8.73 \\
\hline
\end{tabular}

Table 6 System performances under activity- and trip-based approaches.

\begin{tabular}{lcc}
\hline & $\begin{array}{c}\text { Activity-based } \\
\text { approach }\end{array}$ & $\begin{array}{c}\text { Trip-based } \\
\text { approach }\end{array}$ \\
\hline Total travel-time cost in the morning peak (\$) & 9,167 & 28,500 \\
Total travel-time cost in the evening peak $(\$)$ & 12,500 & 28,500 \\
Total schedule-delay cost in the morning peak $(\$)$ & 30,750 & 28,500 \\
Total schedule-delay cost in the evening peak (\$) & 27,500 & 28,500 \\
Total utility of morning home activity $(\$)$ & 314,667 & 311,200 \\
Total utility of evening home activity $(\$)$ & 312,500 & 317,500 \\
Total utility of work activity $(\$)$ & 519,750 & 480,150 \\
Total net utility of the system during a day $(\$)$ & $1,067,000$ & 994,850 \\
\hline
\end{tabular}


Table 7 Effects of flexibility parameter on time allocation and average net utility per commuter during a day.

\begin{tabular}{|c|c|c|c|c|c|c|c|c|c|c|c|}
\hline & \multicolumn{11}{|c|}{ Flexibility parameter $\xi$} \\
\hline $\begin{array}{l}\text { Average queuing time in the } \\
\text { morning peak (h) }\end{array}$ & 0.26 & 0.30 & 0.31 & 0.32 & 0.31 & 0.29 & 0.25 & 0.20 & 0.17 & 0.13 & 0.10 \\
\hline $\begin{array}{l}\text { Average queuing time in the } \\
\text { evening peak (h) }\end{array}$ & 0.46 & 0.42 & 0.38 & 0.35 & 0.30 & 0.26 & 0.21 & 0.16 & 0.14 & 0.14 & 0.16 \\
\hline $\begin{array}{l}\text { Average duration for } \\
\text { morning home activity (h) }\end{array}$ & 7.82 & 7.86 & 7.89 & 7.90 & 7.91 & 7.89 & 7.87 & 7.84 & 7.83 & 7.84 & 7.84 \\
\hline $\begin{array}{l}\text { Average duration for } \\
\text { evening home activity (h) }\end{array}$ & 6.50 & 6.42 & 6.34 & 6.24 & 6.17 & 6.08 & 6.00 & 5.94 & 5.96 & 5.98 & 6.02 \\
\hline $\begin{array}{l}\text { Average duration for work } \\
\text { activity (h) }\end{array}$ & 8.96 & 9.00 & 9.08 & 9.19 & 9.31 & 9.48 & 9.68 & 9.86 & 9.90 & 9.91 & 9.88 \\
\hline $\begin{array}{l}\text { Average net utility per } \\
\text { commuter during a day }(\$)\end{array}$ & 188.72 & 191.12 & 192.69 & 192.97 & 192.43 & 190.67 & 188.03 & 184.27 & 178.96 & 173.25 & 167.05 \\
\hline
\end{tabular}

